\title{
Broadband X-Ray Observations of the 2018 Outburst of the Changing-Look Active Galactic Nucleus NGC 1566
}

\author{
Arghajit Jana, ${ }^{1 \star}$ Neeraj Kumari, ${ }^{1,2}$ Prantik Nandi, ${ }^{3}$ Sachindra Naik, ${ }^{1}$ Arka Chatterjee, ${ }^{4,5}$ Gaurava K. Jaisawal, ${ }^{6}$ \\ Kimitake Hayasaki, ${ }^{5}$ Claudio Ricci ${ }^{7,8}$ \\ ${ }^{1}$ Astronomy \& Astrophysics Division, Physical Research Laboratory, Navrangpura, Ahmedabad, 380009, India \\ ${ }^{2}$ Department of Physics, Indian Institute of Technology, Gandhinagar-382355, Gujarat, India \\ ${ }^{3}$ Department of Astrophysics and Cosmology, S. N. Bose National Centre for Basic Science, Block-JD, Sector-III, Salt Lake, Kolkata, 700106, India \\ ${ }^{4}$ Department of Physics, School of Natural Sciences, UNIST, Ulsan, 44919, Republic of Korea \\ ${ }^{5}$ Department of Astronomy and Space Science, Chungbuk National University, Cheongju, 361-763, Republic of Korea \\ ${ }^{6}$ National Space Institute, Technical University of Denmark, Elektrovej 327-328, DK-2800 Lyngby, Denmark \\ ${ }^{7}$ Núcleo de Astronomía de la Facultad de Ingeniería, Universidad Diego Portales, Av. Ejército Libertador 441, Santiago, Chile \\ ${ }^{8}$ Kavli Institute for Astronomy and Astrophysics, Peking University, Beijing 100871, People's Republic of China
}

Accepted XXX. Received YYY; in original form ZZZ

\begin{abstract}
We study the nature of the changing-look Active Galactic Nucleus NGC 1566 during its June 2018 outburst. During the outburst, the X-ray intensity of the source rises up to $\sim 25-30$ times compared to its quiescent state intensity. We perform timing and spectral analysis of the source during pre-outburst, outburst and post-outburst epochs using semi-simultaneous observations with the XMM-Newton, Nuclear Spectroscopic Telescope Array (NuSTAR) and Neil Gehrels Swift Observatories. We calculate variance, normalized variance, and fractional rms amplitude in different energy bands to study the variability. The broad-band $0.5-70 \mathrm{keV}$ spectra are fitted with phenomenological models, as well as physical models. A strong soft X-ray excess is detected in the spectra during the outburst. The soft excess emission is found to be complex and could originate in the warm Comptonizing region in the inner accretion disc. We find that the increase in the accretion rate is responsible for the sudden rise in luminosity. This is supported by the ' $\mathrm{q}$ '-shape of the hardness-intensity diagram that is generally found in outbursting black hole X-ray binaries. From our analysis, we find that NGC 1566 most likely harbours a low-spinning black hole with the spin parameter $a^{*} \sim 0.2$. We also discuss a scenario where the central core of NGC 1566 could be a merging supermassive black hole.
\end{abstract}

Key words: galaxies: active - galaxies: Seyfert - X-rays: galaxies - X-rays: individual: NGC 1566

\section{INTRODUCTION}

Active galactic nuclei (AGNs) are classified as type-1 or type-2, depending on the presence or absence of broad optical emission lines. The existence of different classes of AGNs can be explained by the unified model (Antonucci 1993), which is based on the orientation of the optically thick torus with respect to our line-of-sight. Recently, a new sub-class of AGNs, known as changing look AGNs (CLAGNs), has been identified by optical observations. These objects display the appearence or disappearance of the broad optical emission lines, transitioning from type-1 (or type 1.2/1.5) to type-2 (or type 1.8/1.9) and vice versa. Several nearby galaxies, such as Mrk 590 (Denney et al. 2014), NGC 2617 (Shappee et al. 2014), Mrk 1018 (Cohen et al. 1986), NGC 7582 (Aretxaga et al. 1999), NGC 3065 (Eracleous \& Halpern 2001), have been found to show such a peculiar behaviour. In the X-rays, a different type of changing-look events have been observed, with AGN switching between Compton-thin (line-ofsight column density, $N_{\mathrm{H}}<1.5 \times 10^{24} \mathrm{~cm}^{-2}$ ) and Compton-thick $\left(\mathrm{CT} ; N_{\mathrm{H}}>1.5 \times 10^{24} \mathrm{~cm}^{-2}\right.$ ) states (Risaliti et al. 2002; Matt et al.

\footnotetext{
^ E-mail: argha@prl.res.in
}

2003). These X-ray changing-look events have been observed in many AGNs, namely, NGC 1365 (Risaliti et al. 2007), NGC 4388 (Elvis et al. 2004), NGC 7582 (Piconcelli et al. 2007; Bianchi et al. 2009), NGC 4395 (Nardini \& Risaliti 2011), IC 751 (Ricci et al. 2016), NGC 4507 (Braito et al. 2013), NGC 6300 (Guainazzi 2002; Jana et al. 2020).

The origin of the CL events is still unclear. The X-ray changinglook events could be explained by variability of the line-of-sight column density $\left(N_{\mathrm{H}}\right)$ associated with the clumpiness of the BLR or of the circumnuclear molecular torus (Nenkova et al. 2008a,b; Elitzur 2012; Yaqoob et al. 2015; Guainazzi et al. 2016; Jana et al. 2020). On the other hand, optical CL events could be related to changes in the accretion rate (Elitzur et al. 2014; MacLeod et al. 2016; Sheng et al. 2017), which could be linked with the appearance and disappearance of the broad line regions (BLRs) (Nicastro 2000; Korista \& Goad 2004; Runnoe et al. 2016). Some of these optical CL events could also be associated with the tidal disruption of a star by the supermassive black hole $(\mathrm{SMBH})$ at the centre of the galaxy (Eracleous et al. 1995; Merloni et al. 2015; Ricci et al. 2020, 2021).

NGC 1566 is a nearby $(\mathrm{z}=0.005)$, face-on spiral galaxy, classified as a type SAB(s)bc (de Vaucouleurs 1973; Shobbrook 1966). 
The AGN was intensively studied over the last 70 years and is one of the first galaxies where variability was detected (Quintana et al. 1975; Alloin et al. 1985, 1986; Winkler 1992). In the 1960s, NGC 1566 was classified as a Seyfert 1, with broad $\mathrm{H} \alpha$ and $\mathrm{H} \beta$ lines (de Vaucouleurs \& de Vaucouleurs 1961). Later, the $\mathrm{H} \beta$ line was found to be weak, leading to the source being classified as a Seyfert 2 (Pastoriza \& Gerola 1970). In the 1970s and 1980s, NGC 1566 was observed to be in the low state with weak $\mathrm{H} \beta$ emission (Alloin et al. 1986). Over the years, it was observed to change its type again from Seyfert 1.9-1.8 to Seyfert 1.5-1.2 (da Silva et al. 2017), with two optical outbursts in 1962 and 1992 (Shobbrook 1966; Pastoriza \& Gerola 1970; da Silva et al. 2017; Oknyansky et al. 2019).

INTEGRAL caught NGC 1566 in the outburst state in hard X-ray band in June 2018 (Ducci et al. 2018). Follow-up observations were carried out in the X-ray, optical, ultraviolet (UV), and infrared (IR) bands (Grupe et al. 2018a; Ferrigno et al. 2018; Kuin et al. 2018; Dai et al. 2018; Cutri et al. 2018). The flux of the AGN was found to increase in all wavebands and reached its peak in July 2018 (Oknyansky et al. 2019, 2020; Parker et al. 2019). Long-term ASASSN and NEOWISE light curves showed that the optical and IR flux started to increase from September 2017 (Dai et al. 2018; Cutri et al. 2018). The Swift/XRT flux increased by about $\sim 25-30$ times (Oknyansky et al. 2019) as the source changed to Seyfert 1.2 from Seyfert 1.8-1.9 type (Oknyansky et al. 2019, 2020). The source became a type-1, with the appearance of strong, broad emission lines (Oknyansky et al. 2019; Ochmann et al. 2020). After reaching their peak, the fluxes declined in all wavebands. After the main outburst, several small flares were observed (Grupe et al. 2018b, 2019).

In this paper, we explore the timing and spectral properties of NGC 1566 during the 2018 outburst using data from the XMMNewton and NUSTAR observatories, covering a broad energy range $(0.5-70 \mathrm{keV})$. In Section 2, we present the observations and describe the procedure adopted for the data extraction. In Section $3 \& 4$, we present results obtained from our timing and spectral analysis, respectively. In Section 5, we discuss our findings. We summarise our results in Section 6.

\section{OBSERVATION AND DATA REDUCTION}

In the present work, we used data from NUSTAR, XMM-Newton and Swift observations of NGC 1566, carried out at different epochs, as reported in Table 1. Out of the eight available epochs, $\mathrm{X} 1$ and $\mathrm{O} 1$ were studied by Parker et al. (2019). We also included those observations for a complete study of the source at different phases (pre-outbursts, outburst and post-outbursts period).

\subsection{NUSTAR}

NUSTAR is a hard X-ray focusing telescopes, consisting of two identical modules: FPMA and FPMB (Harrison et al. 2013). NGC 1566 was observed by NuSTAR six times between 2018 June 26 and 2019 August 21, simultaneously with either XMM-Newton or Swift (see Table 1). Reprocessing of the raw data was performed with the NuSTAR Data Analysis Software (NuSTARDAS, version 1.4.1). Cleaned event files were generated and calibrated by using the standard filtering criteria in the nupipeline task and the latest calibration data files available in the NuSTAR calibration database (CALDB) ${ }^{1}$. The source and background products were extracted

\footnotetext{
${ }^{1}$ http://heasarc.gsfc.nasa.gov/FTP/caldb/data/nustar/fpm/
}

by considering circular regions with radius 60 arcsec and 90 arcsec, respectively. While the source region was centred at the coordinates of the optical counterparts, the background spectrum was extracted from a region devoid of other sources. The spectra and light curves were extracted using the nuproduct task. The light curves were binned at $500 \mathrm{~s}$. We re-binned the spectra with 20 counts per bin by using the grppha task. No background flare was detected in the NUSTAR observations.

\subsection{XMM-Newton}

NGC 1566 was observed by XMM-Newton (Jansen et al. 2001) at five epochs between November 2015 and August 2019. Out of these five observations, the source was observed simultaneously with $\mathrm{NuS}$ TAR in three epochs (see Table 3). We used the Science Analysis System (SAS v16.1. $0^{2}$ ) to reprocess the raw data from EPICpn (Strüder et al. 2001). We considered only unflagged events with PATTERN $\leq 4$. Particle background flares were observed above 10 $\mathrm{keV}$ in X1 and O5. The Good Time Interval (GTI) file was generated considering only intervals with $<0.65$ counts $^{-1}$, using the SAS task tabgtigen. No flares were observed in $\mathrm{O} 1, \mathrm{O} 2$ and $\mathrm{O} 3$. The source and background spectra were initially extracted from a circular region of 30 " centered at the position of the optical counterpart, and from a circular region of 60" radius away from the source, respectively. Then we extracted the spectrum using the SAS task 'especget'. The observed and expected pattern distributions were generated by applying epaplot task on the filtered events. We found that the source spectrum would be affected by photon pile-up and as a result, the expected distributions were significantly discrepant from the observed ones. We therefore considered an annular region of 30 " outer radius and different values of inner radii for the source and checked for the presence of pile-up. We found that, with an inner radius of 10", the source would be pile-up free ${ }^{3}$. We therefore used this inner radius for the spectral extraction. The response files (arf and $r m f$ files) were generated by using SAS tasks ARFGEN and RMFGEN, respectively. Background-subtracted light curves were produced using the FTOOLS task LCMATH. It is also to be noted that we ran SAS task 'correctforpileup' when we generated the pile-up corrected rmf file by using 'rmfgen'.

\subsection{Swift}

Swift monitored NGC 1566 over a decade in both window-timing (WT) and photon counting (PC) modes. The source was observed simultaneously with Swift/XRT and NuSTAR three times (see Table 1 for details). The $0.3-10 \mathrm{keV}$ spectra were generated using the standard online tools provided by the UK Swift Science Data Centre (Evans et al. 2009) ${ }^{4}$. For the present study, we used both WT and PC mode spectra in the $0.3-10 \mathrm{keV}$ range. We also generated long-term light curves in the $0.3-10 \mathrm{keV}$ band using the online-tools ${ }^{5}$. 
Table 1. Log of observations of NGC 1566

\begin{tabular}{|c|c|c|c|c|c|c|c|c|c|c|}
\hline ID & UT Date & NUSTAR & $\operatorname{Exp}(\mathrm{ks})$ & Count s ${ }^{-1}$ & XMM-Newton & $\operatorname{Exp}(\mathrm{ks})$ & Count $\mathrm{s}^{-1}$ & Swift/XRT & $\operatorname{Exp}(\mathrm{ks})$ & Count s ${ }^{-1}$ \\
\hline $\mathrm{X} 1$ & 2015-11-05 & - & - & - & 0763500201 & 91.0 & $1.21 \pm 0.01$ & - & - & - \\
\hline O1 & 2018-06-26 & 80301601002 & 56.8 & $1.99 \pm 0.01$ & 0800840201 & 94.2 & $26.40 \pm 0.02$ & - & - & - \\
\hline $\mathrm{O} 2$ & 2018-10-04 & 80401601002 & 75.4 & $0.55 \pm 0.003$ & 0820530401 & 108.0 & $4.92 \pm 0.01$ & - & - & - \\
\hline $\mathrm{O} 3$ & 2019-06-05 & 80502606002 & 57.2 & $0.19 \pm 0.002$ & 0840800401 & 94.0 & $3.78 \pm 0.01$ & - & - & - \\
\hline $\mathrm{O} 4$ & 2019-08-08 & 60501031002 & 58.9 & $0.60 \pm 0.003$ & - & - & - & 00088910001 & 1.9 & $0.55 \pm 0.02$ \\
\hline O5 & 2019-08-11 & - & - & - & 0851980101 & 18.0 & $4.83 \pm 0.02$ & - & - & - \\
\hline O6 & 2019-08-18 & 60501031004 & 77.2 & $0.33 \pm 0.002$ & - & - & - & 00088910002 & 1.7 & $0.27 \pm 0.01$ \\
\hline $\mathrm{O} 7$ & 2019-08-21 & 60501031006 & 86.0 & $0.36 \pm 0.002$ & - & - & - & 00088910003 & 2.0 & $0.21 \pm 0.01$ \\
\hline
\end{tabular}

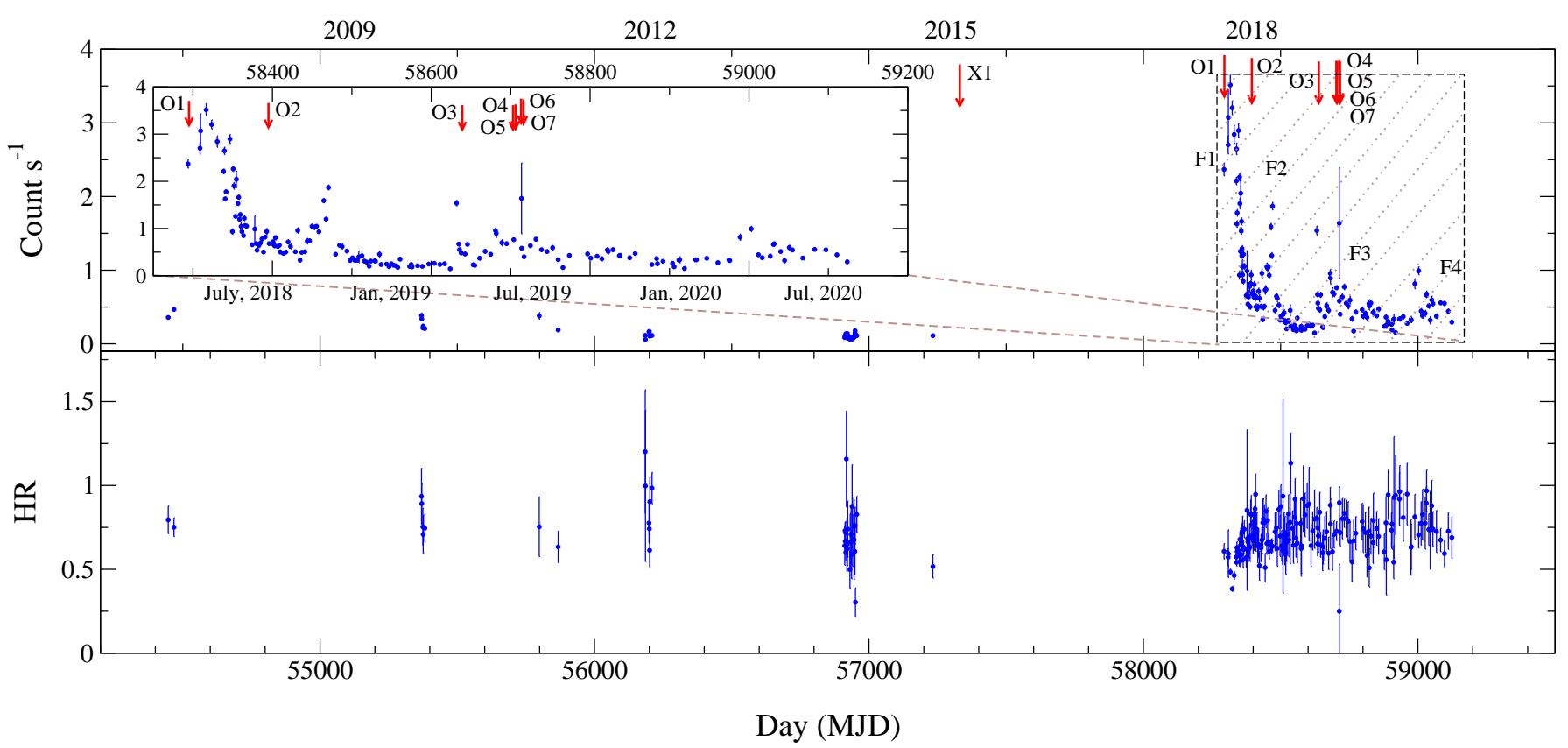

Figure 1. The long-term, $0.3-10 \mathrm{keV} \mathrm{Swift/XRT} \mathrm{light} \mathrm{curve} \mathrm{of} \mathrm{NGC} 1566$ is shown in the top panel. The shaded region shows the light curve between 2018 and 2020. The inset figure in the top panel shows the expanded light curve of the shaded region from 2018 to 2020 for clarity. The red arrows represent the NuSTAR, XMM-Newton and Swift/XRT observations of the source (see Table 1 for details). In the bottom panel, we illustrate the variation of the hardness ratio (HR). The HR is defined as the ratio between count rates in the $1.5-10 \mathrm{keV}$ and the $0.3-1.5 \mathrm{keV}$ band.

\section{RESULTS}

\subsection{Timing Analysis}

We studied the long term Swift/XRT light curves of NGC 1566 in the $0.3-10 \mathrm{keV}$ energy range for the timing analysis. Along with the Swift/XRT light curves, the $0.5-10 \mathrm{keV}$ and $3-70 \mathrm{keV}$ light curves (500 s time-bin) from XMM-Newton and NuSTAR observations were also analysed.

\subsubsection{Outburst Profile}

NGC 1566 was observed intensively with many observatories for about 70 years, starting from the 1950s. In this time period, NGC 1566 showed two major outbursts in optical wavebands, in

\footnotetext{
2 https://www. cosmos.esa.int/web/xmm-newton/sas-threads

3 https://www. cosmos.esa.int/web/xmm-newton/sas-thread-epatplot

4 http://www.swift.ac.uk/user_objects/

5 http://www.swift.ac.uk/user_objects/
}

1962 and 1992, along with several flaring episodes (Alloin et al. 1986; da Silva et al. 2017). Before the major X-ray outburst in 2018, a flaring event was observed in 2010 by the Swift/BAT survey ${ }^{6}$ (Oknyansky et al. 2018, 2019). Since then, NGC 1566 remained in the low state with a luminosity of $L \sim 10^{41} \mathrm{erg} \mathrm{s}^{-1}$ in $2-10 \mathrm{keV}$ energy band.

NGC 1566 was monitored by Swift/XRT over a decade, and it was caught in an outburst in June 2018, when the X-ray intensity increased by $\sim 25-30$ times compared to the quiescent state (Oknyansky et al. 2019). During the 2018 X-ray outburst, the source also brightened in the optical, ultraviolet (UV) and infrared (IR) wavebands (Dai et al. 2018; Cutri et al. 2018). The optical and nearinfrared (NIR) observations showed that the AGN started to brighten since 2017 September (Dai et al. 2018). In the upper panel of Figure 1, we show the long term $0.3-10 \mathrm{keV}$ Swift/XRT light curve. From this figure, it can be seen that the source experienced a major outburst in June 2018 (F1), which was followed by three smaller

${ }^{6}$ https://swift.gsfc.nasa.gov/results/bs $105 \mathrm{mon} / 216$ 
Table 2. Variability in different energy bands are shown here. In some cases, the average error of observational data exceeds the $1 \sigma$ limit, resulting negative excess variance. In such cases, we obtained imaginary $F_{\text {var }}$, which are not included in this table.

\begin{tabular}{|c|c|c|c|c|c|c|c|c|c|c|}
\hline ID & $\begin{array}{c}\text { Energy } \\
(\mathrm{keV}) \\
(2)\end{array}$ & $\begin{array}{l}\mathrm{N} \\
(3)\end{array}$ & $\begin{array}{c}F_{\max } \\
\left(\text { Count } s^{-1}\right) \\
(4)\end{array}$ & $\begin{array}{c}F_{\min } \\
\left(\text { Count } s^{-1}\right) \\
(5)\end{array}$ & $\begin{array}{l}\mathrm{R} \\
\text { (6) }\end{array}$ & $\begin{array}{l}\text { Mean } \\
\text { (7) }\end{array}$ & $\begin{array}{c}\sigma \\
\left(10^{-3}\right) \\
(8)\end{array}$ & $\begin{array}{c}\sigma_{\mathrm{XS}}^{2} \\
\left(10^{-3}\right) \\
(9)\end{array}$ & $\begin{array}{c}\sigma_{\mathrm{NXS}}^{2} \\
\left(10^{-3}\right) \\
(10)\end{array}$ & $\begin{array}{l}F_{\text {var }} \\
(\%) \\
(11)\end{array}$ \\
\hline $\mathrm{X} 1$ & $0.5-3$ & 178 & 1.04 & 0.63 & 1.66 & 0.82 & $71 \pm 2$ & 2.74 & $3.3 \pm 0.7$ & $5.8 \pm 0.6$ \\
\hline $\mathrm{X} 1$ & $3-10$ & 178 & 0.19 & 0.07 & 2.63 & 0.12 & $19 \pm 1$ & - & - & - \\
\hline $\mathrm{O} 1$ & $0.5-3$ & 185 & 27.84 & 16.69 & 1.67 & 21.89 & $2573 \pm 78$ & 6.55 & $13.7 \pm 0.2$ & $11.7 \pm 0.6$ \\
\hline $\mathrm{O} 1^{*}$ & $3-10$ & 145 & 2.35 & 1.31 & 1.80 & 1.81 & $210 \pm 11$ & 2.2 & $9.7 \pm 1.0$ & $9.8 \pm 0.8$ \\
\hline $\mathrm{O} 1$ & $10-70$ & 145 & 1.21 & 0.54 & 2.23 & 0.80 & $98 \pm 5$ & 4.94 & $7.8 \pm 1.5$ & $8.8 \pm 1.0$ \\
\hline $\mathrm{O} 2$ & $0.5-3$ & 213 & 5.69 & 2.98 & 1.91 & 4.05 & $628 \pm 15$ & 380.2 & $93.9 \pm 0.6$ & $30.6 \pm 0.8$ \\
\hline $\mathrm{O} 2^{*}$ & $3-10$ & 197 & 0.86 & 0.29 & 2.99 & 0.52 & $98 \pm 3$ & 6.50 & $24.4 \pm 2.6$ & $15.6 \pm 1.2$ \\
\hline $\mathrm{O} 2$ & $10-70$ & 197 & 0.47 & 0.12 & 3.78 & 0.25 & $51 \pm 2$ & 1.11 & $17.2 \pm 0.4$ & $13.1 \pm 1.6$ \\
\hline $\mathrm{O} 3$ & $0.5-3$ & 185 & 3.74 & 2.52 & 1.48 & 3.13 & $289 \pm 11$ & 72.2 & $23.1 \pm 0.5$ & $15.2 \pm 0.4$ \\
\hline $\mathrm{O}^{*}$ & $3-10$ & 161 & 0.48 & 0.08 & 6.46 & 0.22 & $48 \pm 2$ & 0.16 & $3.2 \pm 5.4$ & $5.7 \pm 4.8$ \\
\hline $\mathrm{O} 3$ & $10-70$ & 161 & 0.47 & 0.04 & 12.5 & 0.16 & $45 \pm 2$ & 0.28 & $11.2 \pm 0.9$ & $10.6 \pm 4.3$ \\
\hline $\mathrm{O} 4$ & $3-10$ & 145 & 0.99 & 0.36 & 2.78 & 0.55 & $86 \pm 3$ & 4.76 & $15.4 \pm 2.2$ & $12.4 \pm 1.1$ \\
\hline $\mathrm{O} 4$ & $10-70$ & 145 & 0.37 & 0.15 & 2.42 & 0.26 & $40 \pm 1$ & 0.39 & $5.9 \pm 2.8$ & $7.7 \pm 1.8$ \\
\hline O5 & $0.5-3$ & 27 & 3.71 & 3.33 & 1.12 & 3.52 & $109 \pm 12$ & 0.46 & $0.1 \pm 0.3$ & $0.6 \pm 2.2$ \\
\hline O5 & $3-10$ & 27 & 0.55 & 0.15 & 3.68 & 0.39 & $116 \pm 3$ & 10.78 & $71.5 \pm 14.7$ & $26.7 \pm 4.6$ \\
\hline O6 & $3-10$ & 191 & 0.45 & 0.16 & 2.77 & 0.31 & $52 \pm 2$ & 1.27 & $13.1 \pm 2.6$ & $11.4 \pm 1.3$ \\
\hline O6 & $10-70$ & 191 & 0.23 & 0.08 & 2.83 & 0.16 & $30 \pm 1$ & 0.12 & $4.9 \pm 3.8$ & $7.0 \pm 02.7$ \\
\hline O7 & $3-10$ & 208 & 1.46 & 0.21 & 6.85 & 0.35 & $98 \pm 7$ & 2.50 & $20.3 \pm 7.4$ & $14.3 \pm 2.7$ \\
\hline $\mathrm{O} 7$ & $10-70$ & 208 & 0.73 & 0.09 & 8.22 & 0.17 & $53 \pm 4$ & - & - & - \\
\hline
\end{tabular}

Columns in the table represent - (1) ID of observation, (2) energy range, (3) number of data points or length of the light curve, (4) maximum count of the light curve, (5) minimum count of the light curve, (6) ratio of maximum count to minimum count, $R=F_{\max } / F_{\min }$, (7) mean count of the light curve, (8) standard deviation of the light curve, (9) excess variance, (10) normalized excess variance, (11) fractional rms amplitude of the light curve. *During O1, O2 and O3, we reported only NUSTAR observation in $3-10 \mathrm{keV}$ energy range, although both XMM-Newton and NuSTAR data were available in $3-10 \mathrm{keV}$ energy band.

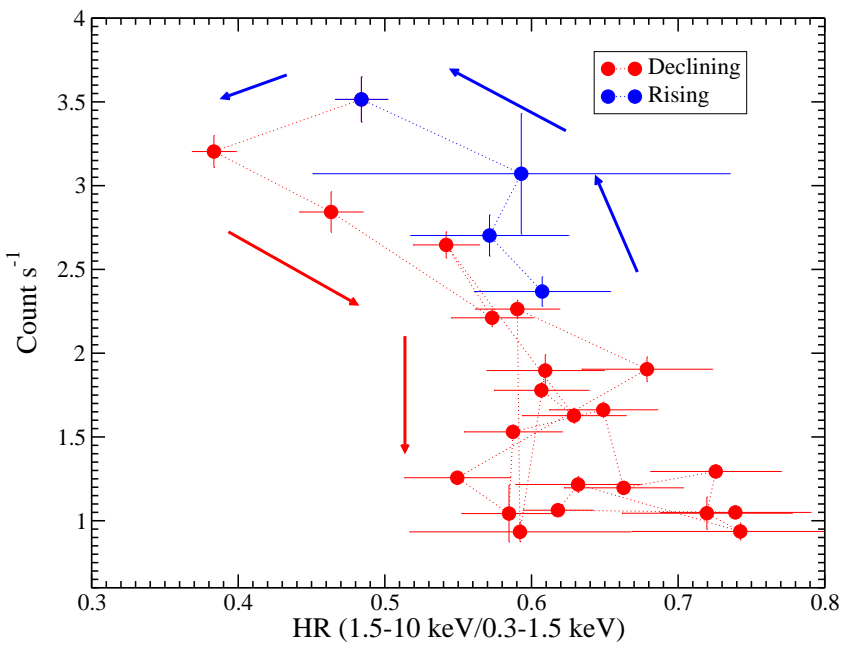

Figure 2. Hardness-intensity diagram (HID) for the first outburst F1 (from MJD 58308 to MJD 58380). The $0.3-10 \mathrm{keV}$ Swift/XRT count rate is plotted as a function of hardness ratio (HR). The arrow marks in the figure represent the evolution of the outburst.

flaring events in December 2018 (F2; Grupe et al. 2018b), May 2019 (F3; Grupe et al. 2019), and May 2020 (F4). The smaller outbursts (F2, F3 and F4) were not as bright as the main outburst (F1). In the bottom panel of Figure 1, we show the evolution of the hardness ratio (HR; i.e. the ratio between the $1.5-10 \mathrm{keV}$ and $0.3-1.5 \mathrm{keV}$ count rate) with time. Unlike the Swift/XRT light curve (top panel), the HR plot did not show any significant long-term variability. In Figure 2, we show hardness-intensity diagram (Homan et al. 2001; Remillard \& McClintock 2006) for the main outburst (F1), where the Swift/XRT count rate is plotted as a function of HR. The HID or 'q'-diagram appeared to show a 'q'-like shape, which is ubiquitous for outbursting Galactic black hole X-ray binaries. Interestingly, we did not observe any clear sign of ' $q$ '-shape HID for the next three recurrent outbursts.

\subsubsection{Variability}

We studied the source variability in different energy bands. As the soft excess in AGNs is generally observed below $3 \mathrm{keV}$, whereas the primary emission is observed above $3 \mathrm{keV}$, we analysed the $X M M$ Newton light curves in $0.5-3 \mathrm{keV}$ and $3-10 \mathrm{keV}$ energy ranges. We also studied the NUSTAR light curves in two separate bands $(3-10 \mathrm{keV}$ and $10-70 \mathrm{keV})$.

We calculated the peak-to-peak ratio of the light curves, which is defined as $R=F_{\max } / F_{\min }$, where $F_{\max }$ and $F_{\min }$ are the maximum and minimum count rates, respectively. The light curves in different energy bands $(0.5-3 \mathrm{keV}, 3-10 \mathrm{keV}$, and $10-70 \mathrm{keV})$ showed different magnitude of variability. In all observations, $R$ was higher in the $3-10 \mathrm{keV}$ energy band than in the $0.5-3 \mathrm{keV}$ range. In the $10-70 \mathrm{keV}$ energy band, $R$ was higher than in the lower energy band, except for $\mathrm{O} 4$. The mean value of $R$ in $0.5-3 \mathrm{keV}, 3-10 \mathrm{keV}$, and $10-70 \mathrm{keV}$ energy bands are $\langle R\rangle=1.34,3.43$, and 5.33, 
respectively. Thus, it is clear that the light curves showed higher variability in the higher energy bands in terms of R. However, this is too simplistic as very high or low count could be generated due to systematic/instrumental error. Hence, we calculated the normalized variance $\left(\sigma_{\mathrm{NXS}}^{2}\right)$ and fractional variability ( $F_{\text {var }}$ to study the variability.

We calculated the fractional variability $\left(F_{\text {var }}\right)$ (Edelson et al. 1996, 2001; Edelson \& Malkan 2012; Nandra et al. 1997; Vaughan et al. 2003 ) in the $0.5-3,3-10$ and $10-70 \mathrm{keV}$ bands for a light curve of $x_{i}$ counts $s^{-1}$ with uncertainties $\sigma_{i}$ of length $N$, with a mean $\mu$ and standard deviation $\sigma$ is given by,

$F_{\mathrm{var}}=\sqrt{\frac{\sigma_{\mathrm{XS}}^{2}}{\mu^{2}}}$,

where, $\sigma_{\mathrm{XS}}^{2}$ is the excess variance (Nandra et al. 1997; Edelson et al. 2002) which is given by,

$\sigma_{\mathrm{XS}}^{2}=\sigma^{2}-\sigma_{\mathrm{err}}^{2}$

where, $\sigma_{\mathrm{err}}^{2}$ is the mean squared error. The $\sigma_{\mathrm{err}}^{2}$ is given by,

$\sigma_{\mathrm{err}}^{2}=\frac{1}{N} \sum_{i=1}^{N} \sigma_{\mathrm{i}}^{2}$

The normalized excess variance is given by,

$\sigma_{\mathrm{NXS}}^{2}=\frac{\sigma_{\mathrm{XS}}^{2}}{\mu^{2}}$.

The uncertainties in $F_{\mathrm{var}}$ and $\sigma_{\mathrm{NXS}}$ (Vaughan et al. 2003) are given by,

$\operatorname{err}\left(F_{\mathrm{var}}\right)=\sqrt{\left(\sqrt{\frac{1}{2 N}} \frac{\sigma_{\mathrm{err}}^{2}}{\mu^{2} F_{\mathrm{var}}}\right)^{2}+\left(\frac{1}{\mu} \sqrt{\frac{\sigma_{\mathrm{err}}^{2}}{N}}\right)^{2}}$,

and

$\operatorname{err}\left(\sigma_{\mathrm{NXS}}^{2}\right)=\sqrt{\left(\sqrt{\frac{2}{N}} \frac{\sigma_{\mathrm{err}}^{2}}{\mu^{2}}\right)^{2}+\left(\sqrt{\frac{\sigma_{\mathrm{err}}^{2}}{N}} \frac{2 F_{\mathrm{var}}}{\mu}\right)^{2}}$.

For a few observations, we could not estimate excess variance due to large errors in the data. Thus, from the normalized variance $\left(\sigma_{\mathrm{NXS}}^{2}\right)$, the trend of variability was not clear. We calculated the fractional rms variability amplitude $\left(F_{\mathrm{var}}\right)$ to study the variability. We obtained the highest fractional variability $\left(F_{\text {var }}\right)$ in the $0.5-3 \mathrm{keV}$ energy range for four observations (X1, O1, O2 \& O3), while the highest variability is observed in $3-10 \mathrm{keV}$ range for $\mathrm{O}$. The mean value of the fractional variability in $0.5-3 \mathrm{keV}, 3-10 \mathrm{keV}$, and $10-$ $70 \mathrm{keV}$ energy ranges were $\left\langle F_{\mathrm{var}}\right\rangle=12.8 \pm 0.5 \%, 12.0 \pm 2.8 \%$, and $7.9 \pm 2.4 \%$, respectively. This indicates that the strongest variability is observed in the $0.5-3 \mathrm{keV}$ energy range. The variability parameters discussed here are reported in Table 2.

\subsubsection{Correlation}

The soft excess is generally observed below $3 \mathrm{keV}$. To investigate the origin of the soft excess, we calculated the time delay between
Table 3. ZDCF results.

\begin{tabular}{|c|c|c|c|}
\hline ID & $\begin{array}{c}\text { soft excess } \\
\qquad t^{\dagger} \\
(\mathrm{min})\end{array}$ & $\begin{array}{c}\sigma^{\dagger} \\
(\min )\end{array}$ & $\mathrm{amp}^{\dagger}$ \\
\hline $\mathrm{X} 1$ & $-10.5 \pm 13.0$ & $145.2 \pm 8.3$ & $0.24 \pm 0.07$ \\
\hline $\mathrm{O} 1$ & $13.8 \pm 3.1$ & $103.3 \pm 8.3$ & $0.84 \pm 0.08$ \\
\hline $\mathrm{O} 2$ & $11.7 \pm 5.9$ & $222.9 \pm 8.3$ & $0.90 \pm 0.07$ \\
\hline $\mathrm{O} 3$ & $13.9 \pm 7.8$ & $263.4 \pm 8.3$ & $0.69 \pm 0.07$ \\
\hline O5 & - & - & - \\
\hline
\end{tabular}

ZDCF correlation between primary X-ray continuum (3 - 10 energy band) and soft excess $(0.5-3 \mathrm{keV}$ energy band). $\sigma$ 's and amp's are FWHM and amplitude of the ZDCF function. Note that the maximum amplitude can be 1 .

the soft X-ray photons $(0.5-3 \mathrm{keV}$ range $)$ and the continuum photons $(3-10 \mathrm{keV})$ using cross-correlation method from the $X M M$ Newton observations. We used the $\xi$-transformed discrete correlation function (ZDCF) method (Alexander 1997) ${ }^{7}$ to investigate the timedelay between the soft-excess and the X-ray continuum. The ZDCF co-efficient was calculated for two cases: omitting the zero lag points and including the zero lag points. In both cases, similar results were obtained. A strong correlation between the $0.5-3 \mathrm{keV}$ and $3-10 \mathrm{keV}$ energy bands was observed (amplitude $>0.7$ ) during observations $\mathrm{O} 1, \mathrm{O} 2$ and $\mathrm{O} 3$. However, no significant delay was observed during those observations. The values of ZDCF coefficient with time delay are presented in Table 3 .

\subsection{Spectral Analysis}

We carry out the X-ray spectral analysis using data obtained from the Swift/XRT, XMM-Newton and NuSTAR observations of NGC 1566 using XSPEC v12.10 package (Arnaud 1996) ${ }^{8}$. The spectral analysis was performed using simultaneous $X M M$ Newton and NUSTAR observations in the $0.5-70 \mathrm{keV}$ energy band for three epochs, simultaneous Swift/XRT and NuSTAR observations $(0.5-70 \mathrm{keV})$ for three epochs, and XMM-Newton observations for two epochs $(0.5-10 \mathrm{keV})$, between 2015 November 5 and 2019 August 21 (see Table 1).

For the spectral analysis, we used various phenomenological and physical models, namely, powerlaw (PL) ${ }^{9}$, NTHCOMP ${ }^{10}$ (Zdziarski et al. 1996; Życki et al. 1999), OPTXAGNF ${ }^{11}$ (Done et al. 2012), and RELXILL ${ }^{12}$ (García et al. 2014; Dauser et al. 2014) to understand the X-ray properties of NGC 1566. In general, an X-ray spectrum of an AGN consists of a power-law continuum, a reflection hump at around $15-40 \mathrm{keV}, \mathrm{a} \mathrm{Fe} \mathrm{K} \alpha$ fluorescent line, and a soft X-ray component below $2 \mathrm{keV}$ (Netzer 2015; Padovani et al. 2017; Ricci et al. 2017). The observed X-ray emission also suffers from absorption caused by the interstellar medium and the torus. In our analysis, we used two absorption components. For the Galactic interstellar absorption, we used TBabs ${ }^{13}$ (Wilms et al. 2000) with fixed hydrogen column density of $N_{\mathrm{H}}=7.15 \times 10^{19}$ $\mathrm{cm}^{-2}$ (HI4PI Collaboration et al. 2016). In addition, we also used a

\footnotetext{
7 http://www.weizmann.ac.il/particle/tal/research-activities/software

8 https://heasarc.gsfc.nasa.gov/xanadu/xspec/

9 https://heasarc.gsfc.nasa.gov/xanadu/xspec/manual/node213.html

10 https://heasarc.gsfc.nasa.gov/xanadu/xspec/manual/node205.html

11 https://heasarc.gsfc.nasa.gov/xanadu/xspec/manual/node206.html

12 www.sternwarte.uni-erlangen.de/ dauser/research/relxill/

13 https://heasarc.gsfc.nasa.gov/xanadu/xspec/manual/node265.html
} 

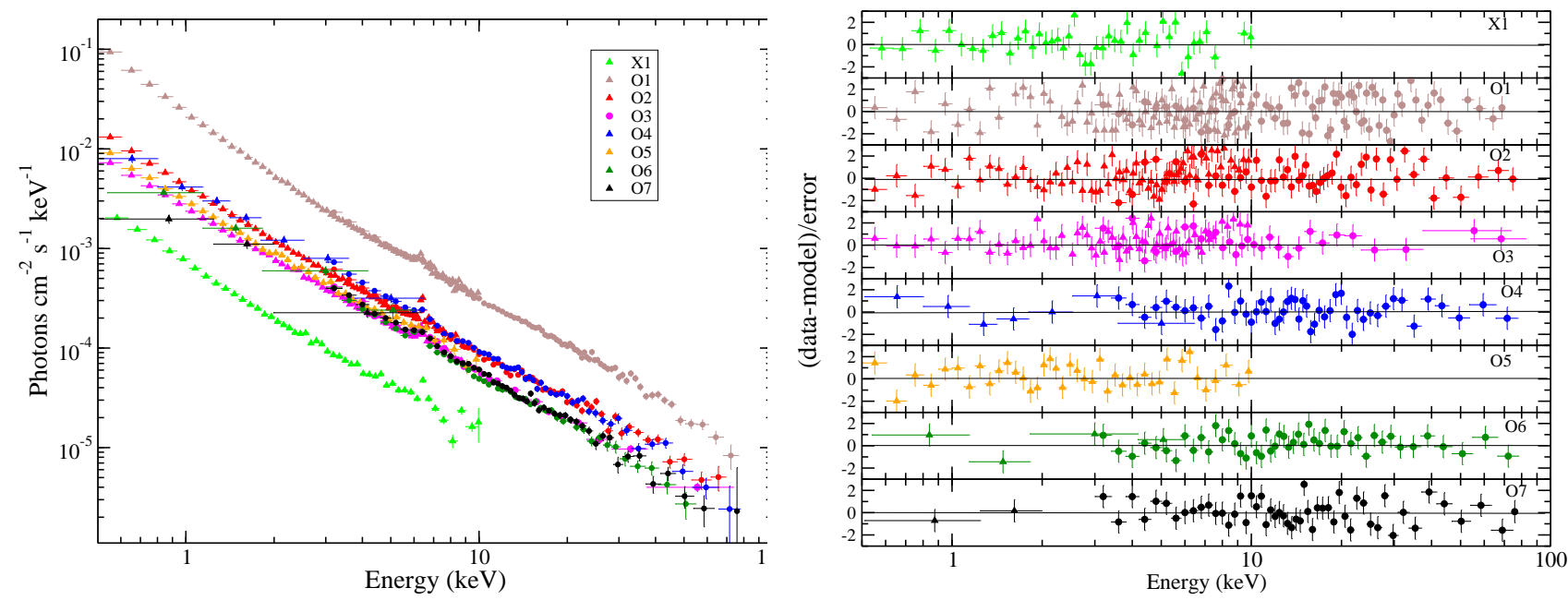

Figure 3. The left panel shows the unfolded spectra obtained from each observations. Triangles represent the XMM-Newton or Swift/XRT data, while circles represent the NUSTAR data. The light-green, brown, red, magenta, blue, orange, dark green and black points represent the observation X1, O1, O2, O3, O4, O5, $\mathrm{O} 6$ and $\mathrm{O} 7$, respectively. The residuals obtained after fitting the source spectra with Model-1 and Model-3 are shown in the right panels. The spectra are re-binned for visual purpose.

ionized absorption model zxipcf. For both the absorption components, we used the wilms abundances (Wilms et al. 2000) and the Verner cross-section (Verner et al. 1996). In this work, we used the following cosmological parameters : $H_{0}=70 \mathrm{~km} \mathrm{~s}^{-1} \mathrm{Mpc}^{-1}$, $\Lambda_{0}=70$, and $\sigma_{M}=0.27$ (Bennett et al. 2003). The uncertainties in each spectral parameters are calculated using the 'error' command in XSPEC, and reported at the $90 \%$ confidence $(1.6 \sigma)$.

\subsubsection{Model 1: POWERLAW}

We built our baseline spectral model with power-law continuum, along with the soft-excess, and Fe K-line emission. We used a blackbody component (bbody in XSPEC) for the soft-excess, and one or more Gaussian functions to incorporate the Fe K complex. Out of the eight epochs, two Gaussian lines were required for six observations. Two ionized absorbers were also needed while fitting the data from three observations. The final model (hereafter Model-1) reads in XSPEC as,

TB $\times$ zxipcf1 $\times$ zxipcf2 $\times($ zPL1 $+z G a+z G a+$ bbody).

We started our analysis with the pre-outburst XMM-Newton observation $\mathrm{X} 1$ (in the $0.5-10 \mathrm{keV}$ energy range), $\sim 2.5$ years prior to the 2018 outburst. Model-1 provided a good fit to the XMMNewton data, with $N_{\mathrm{H}}=(3.53 \pm 0.06) \times 10^{21} \mathrm{~cm}^{-2}$ and power-law photon index of $\Gamma=1.72 \pm 0.05$, with $\chi^{2}=1073$ for 998 degrees of freedom (dof). Along with this, an iron $\mathrm{K} \alpha$ emission line at $\sim 6.4 \mathrm{keV}$ with an equivalent width $(\mathrm{EW})$ of $\sim 206_{-18}^{+4} \mathrm{eV}$ was detected.

Next, we analyzed simultaneous observations of NGC 1566 with XMM-Newton and NUSTAR in the rising phase of the 2018 outburst (O1). First, we included one zxipcf component in our spectral model. The fit returned with $\chi^{2}=2850$ for 2562 degrees of freedom (dof). However, negative residuals were clearly observed in soft $\mathrm{X}$-rays $(<1 \mathrm{keV})$. Thus, we included another zxipcf component, and our fit improved significantly with $\Delta \chi^{2}=106$ for 3 dof. The spectral fitting in $0.5-70 \mathrm{keV}$ range returned $\Gamma=1.85 \pm 0.04$, and $\chi^{2}=2744$ for 2559 dof. The Fe $\mathrm{K} \alpha$ line was detected at $6.38 \mathrm{keV}$ with EW of $114 \pm 15 \mathrm{eV}$, as well as another emission feature at $6.87 \mathrm{keV}$, with $\mathrm{EW}<37 \mathrm{eV}$. This line could be associated with Fe XXVI line. We required two ionized absorber to fit the spectra, one low-ionization absorber $\left(\xi \sim 10^{1.7 \pm 0.1}\right)$ with $N_{\mathrm{H}, 1}=(8.1 \pm 2.2) \times 10^{20} \mathrm{~cm}^{-2}$, and one high-ionization absorber $\left(\xi \sim 10^{4.7 \pm 0.4}\right)$ with $N_{\mathrm{H}, 2}=(4.3 \pm 0.4) \times 10^{21} \mathrm{~cm}^{-2}$. The highionizing absorber required a high covering fraction $(C F>0.73)$, while the low-ionizing absorber a moderate covering fraction with $C F \sim 0.46 \pm 0.04$.

The next observation $(\mathrm{O} 2)$ was carried out on 2018 October 10, simultaneously with XMM-Newton and NUSTAR, covering the $0.5-$ $70 \mathrm{keV}$ energy range. The source was in the decay phase of the outburst at the time of the observation. We started our fitting with one zxipcf component. Although, the model provided a good fit to the data with $\chi^{2}=2298$ for 2130 dof, an absorption feature was seen in the residuals. Thus, we added a second zxipcf component, and our fit returned with $\chi^{2}=2224$ for 2127 dof. The photon index decreased marginally compared to $\mathrm{O} 1(\Gamma=1.78 \pm 0.02)$, while the column density increased slightly for both low and high-ionizing absorbers. The $\mathrm{Fe} \mathrm{K} \alpha$ and $\mathrm{Fe}$ XXVI lines were detected at $6.41 \mathrm{keV}$ and 6.89 $\mathrm{keV}$, with EWs of $126_{-21}^{+3} \mathrm{eV}$ and $<49 \mathrm{eV}$, respectively. The next simultaneous observations of NGC 1566 with XMM-Newton and NUSTAR (O3) were carried out $\sim 8$ months after the end of the outburst. The source was in a low state during the observation. Similar to $\mathrm{O} 1$ and $\mathrm{O} 2$, adding second absorption component improved the fit $\Delta \chi^{2}=67$ for 3 dof. The column density increased to $N_{\mathrm{H}, 1}=$ $(1.24 \pm 0.14) \times 10^{21} \mathrm{~cm}^{-2}$ for the low-ionization absorber, while the column density decreased to $N_{\mathrm{H}, 2}=(8.9 \pm 0.2) \times 10^{20} \mathrm{~cm}^{-2}$ for the high-ionization absorber. The photon index was found to be $\Gamma=$ $1.68 \pm 0.02$ in this observation. We detected both $\mathrm{Fe} \mathrm{K} \alpha$ and $\mathrm{Fe} \mathrm{k} \beta$ lines at $6.39 \mathrm{keV}$ and $7.04 \mathrm{keV}$, with EWs of $117 \pm 14 \mathrm{eV}$ and $<92 \mathrm{eV}$, respectively.

The last four observations (O4, O5, O6 \& O7) were carried out in the span of 13 days. Observation $\mathrm{O} 4$ was made during the second small flare (F2), after the 2018 main outburst. During these four observations, the photon index was nearly constant $(\Gamma \sim 1.67 \pm$ $0.07-1.69 \pm 0.06$ ), while the column density of the low-ionizing absorber was found to vary in the range of $N_{\mathrm{H}, 1} \sim 1.2-1.3 \times 10^{21}$ $\mathrm{cm}^{-2}$. A high-ionization absorber was not required to fit the spectra of these four observations. We also observed a low covering fraction in these four observations (see Table 4). The $\mathrm{Fe} \mathrm{K} \alpha$ line was detected in all four observations, with EW $>100 \mathrm{eV}$ (except O5). During our 

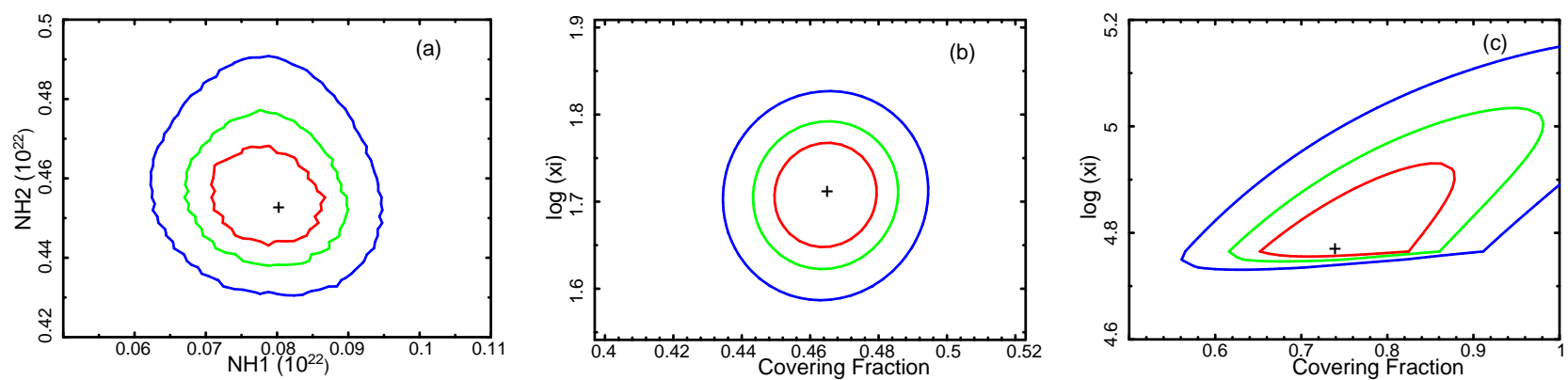

Figure 4. Left panel shows the $2 \mathrm{D}$ contour between the column density of the low-ionizing absorber $\left(N_{\mathrm{H}, 1}\right)$ and high-ionizing absorber $\left(N_{\mathrm{H}, 2}\right)$ for $\mathrm{O} 1$. The middle and right panel show 2D contour between $\log \xi$ and covering fraction for low and high-ionizing absorber, respectively.

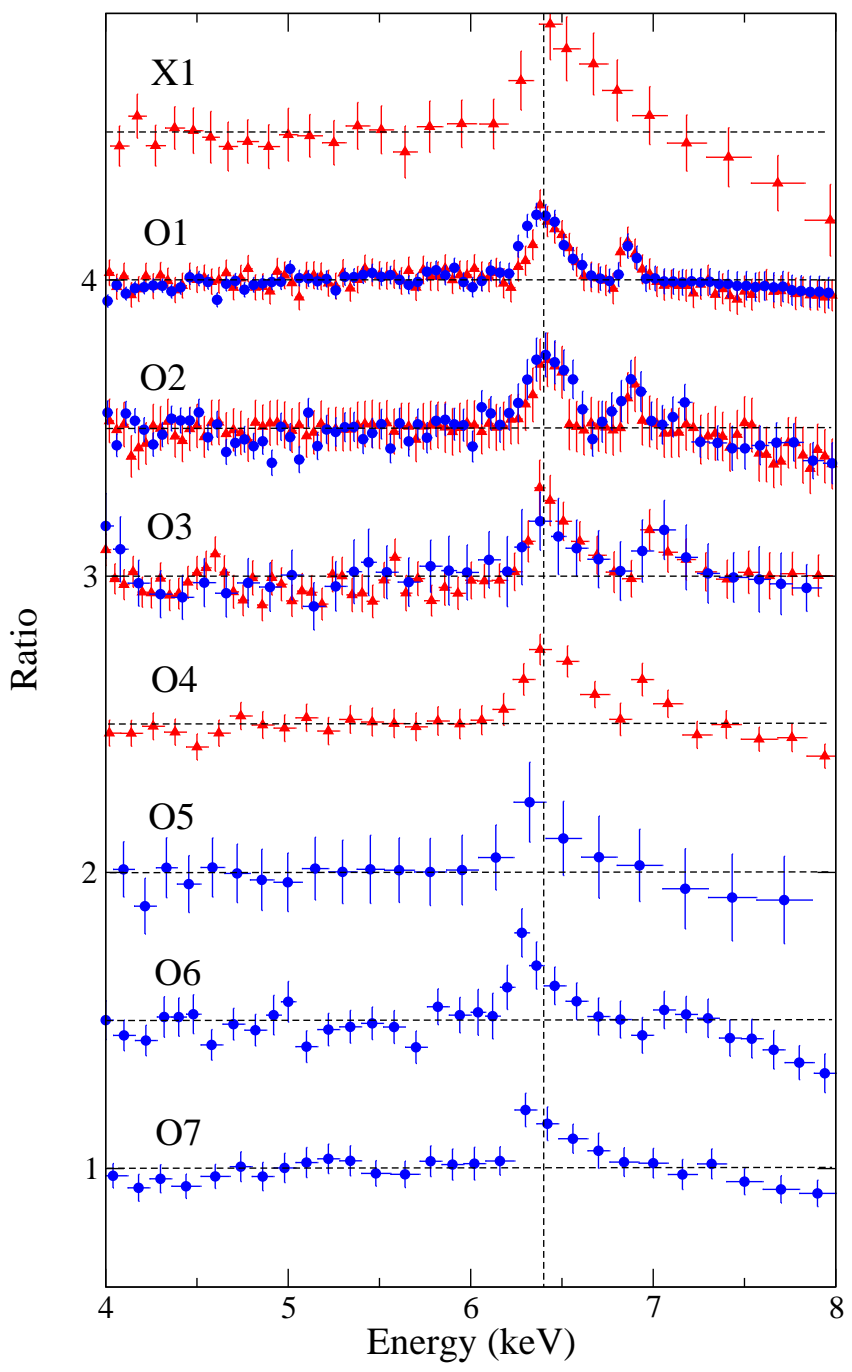

Figure 5. Evolution of Fe complex. The ratio of XMM-Newton and $N u S$ $T A R$ data to the power-law continuum model for every observations are shown. The red triangles and blue circles represent the ratio obtained from XMM-Newton and NUSTAR data, respectively. The vertical dashed line represent $\mathrm{E}=6.4 \mathrm{keV}$. The horizontal dashed lines represent the data/model=1 for each observation. The ratios are re-scaled by adding 0.5 in the $y$-axis separating the observations. observations, the blackbody temperature $\left(T_{\mathrm{bb}}\right)$ was observed to be remarkably constant with $T_{\mathrm{bb}} \sim 110 \mathrm{eV}$. The parameters obtained by our spectral fitting results are listed in Table 4. Model-1 fitted spectra of NGC 1566 are shown in the left panel of Fig. 3, whereas the corresponding residuals are shown in the right panels. To test for the presence of degeneracies between the column densities of two ionizing absorbers, we plotted the $2 \mathrm{D}$ contour in Fig. 4 a for the observation O1. In Fig. 4b and Fig. 4c, we show 2D contours between $\log (\xi)$ and covering fraction for the low and high-ionizing absorbers, respectively, for observation O1. In Fig 5, we show the residuals above the continuum in $4-8 \mathrm{keV}$ energy range for Fe line emission. In Fig. 6a, we also show the unfolded spectrum fitted with Model-1 for observation $\mathrm{O} 2$.

\subsubsection{Model 2: NTHCOMP}

While fitting the source spectra with Model-1 provided us with information on the spectral shape and hydrogen column density, it provided limited information on the physical properties of the Comptonizing plasma. The Comptonizing plasma can be characterized by the electron temperature $\left(k T_{\mathrm{e}}\right)$ and optical depth $(\tau)$. In order to understand the properties of the Compton cloud, we replaced the powerlaw continuum model with NTHCOMP (Zdziarski et al. 1996; Życki et al. $1999)$ in Model-1. The NTHCOMP model provided us the photon index $(\Gamma)$ and the hot electron temperature of the Compton cloud $\left(k T_{\mathrm{e}}\right)$. The optical depth can be easily calculated from the information on $\Gamma$ and $k T_{\mathrm{e}}$ using the following equation (Sunyaev \& Titarchuk 1980; Zdziarski et al. 1996),

$\tau \sim \sqrt{\frac{9}{4}+\frac{m_{e} c^{2}}{k T_{\mathrm{e}}} \frac{3}{(\Gamma-1)(\Gamma+2)}}-\frac{3}{2}$.

This model (hereafter Model-2) reads in XSPEC as,

TB $\times$ zxipcf1 $\times$ zxipcf $2 \times(\mathrm{NTHCOMP}+\mathrm{zGa}+\mathrm{zGa}+$ bbody).

We fixed the seed photon temperature at $k T_{\mathrm{S}}=30 \mathrm{eV}$, which is reasonable for a BH of mass $\sim 8.3 \times 10^{6} M_{\odot}$ (Shakura \& Sunyaev 1973; Makishima et al. 2000). We required two absorption component during $\mathrm{O} 1, \mathrm{O} 2$ and $\mathrm{O} 3$. Inclusion of second absorption improved the fit significantly with $\Delta \chi^{2}=108,84,78$ for 3 dof, during O1, O2 and $\mathrm{O} 3$, respectively. The photon indices and column densities obtained are similar to those obtained using Model-1. We found that $k T_{\mathrm{e}}=102 \pm 5 \mathrm{keV}$ for observation X1. During the rising phase of the outburst (observation O1), the Compton cloud was found to be relatively cooler, with $k T_{\mathrm{e}}=61 \pm 7 \mathrm{keV}$. The Compton cloud was hot during the later observations, with the electron temperature increasing up to $106 \pm 7 \mathrm{keV}$ within the eight observations analyzed here. A 
Table 4. Best-fit parameters obtained from the spectral fitting of the source spectra with Model-1 (POWER-LAW) \& Model-2 (NTHCOMP).

\begin{tabular}{|c|c|c|c|c|c|c|c|c|c|}
\hline & & $\mathrm{X} 1$ & $\mathrm{O} 1$ & $\mathrm{O} 2$ & $\mathrm{O} 3$ & $\mathrm{O} 4$ & O5 & O6 & $\mathrm{O} 7$ \\
\hline \multicolumn{2}{|c|}{$N_{\mathrm{H}, 1}\left(10^{21} \mathrm{~cm}^{-2}\right)$} & $3.53_{-0.06}^{+0.05}$ & $0.81_{-0.22}^{+0.13}$ & $0.96_{-0.18}^{+0.15}$ & $1.24_{-0.11}^{+0.14}$ & $1.33_{-0.10}^{+0.14}$ & $1.18_{-0.09}^{+0.08}$ & $1.25_{-0.16}^{+0.12}$ & $1.30_{-0.22}^{+0.17}$ \\
\hline \multicolumn{2}{|l|}{$\log \xi_{1}$} & $-3^{\dagger}$ & $1.71_{-0.11}^{+0.12}$ & $1.81_{-0.08}^{+0.10}$ & $1.37_{-0.09}^{+0.08}$ & $1.10_{-0.08}^{+0.04}$ & $0.26_{-0.05}^{+0.09}$ & $0.17_{-0.05}^{+0.14}$ & $0.21_{-0.08}^{+0.03}$ \\
\hline \multicolumn{2}{|c|}{ Cov Frac1 } & $0.20_{-0.05}^{+0.13}$ & $0.46_{-0.03}^{+0.04}$ & $0.31_{-0.04}^{+0.09}$ & $0.33_{-0.12}^{+0.07}$ & $<0.12$ & $0.24_{-0.15}^{+0.12}$ & $0.17_{-0.08}^{+0.04}$ & $<0.1$ \\
\hline \multicolumn{2}{|c|}{$N_{\mathrm{H}, 2}\left(10^{21} \mathrm{~cm}^{-2}\right)$} & - & $4.31_{-0.26}^{+0.41}$ & $4.56_{-0.47}^{+0.58}$ & $0.89_{-0.17}^{+0.22}$ & - & - & - & - \\
\hline \multicolumn{2}{|l|}{$\log \xi_{2}$} & - & $4.73_{-0.02}^{+0.40}$ & $3.56_{-0.08}^{+0.26}$ & $3.07_{-0.07}^{+0.19}$ & - & - & - & - \\
\hline \multicolumn{2}{|c|}{ Cov Frac2 } & - & $>0.73$ & $0.61_{-0.21}^{+0.26}$ & $0.54_{-0.39}^{+0.23}$ & - & - & - & - \\
\hline \multicolumn{2}{|l|}{$\Gamma$} & $1.72_{-0.05}^{+0.05}$ & $1.85_{-0.04}^{+0.04}$ & $1.78_{-0.02}^{+0.02}$ & $1.68_{-0.02}^{+0.02}$ & $1.67_{-0.07}^{+0.05}$ & $1.68_{-0.04}^{+0.03}$ & $1.69_{-0.06}^{+0.04}$ & $1.67_{-0.07}^{+0.05}$ \\
\hline \multicolumn{2}{|c|}{ PL Norm $\left(10^{-3}\right)$} & $1.59_{-0.18}^{+0.16}$ & $14.6_{-2.05}^{+1.43}$ & $5.97_{-0.10}^{+0.06}$ & $2.45_{-0.10}^{+0.05}$ & $4.71_{-0.08}^{+0.07}$ & $2.84_{-0.09}^{+0.13}$ & $2.78_{-0.15}^{+0.11}$ & $2.58_{-0.17}^{+0.10}$ \\
\hline \multirow[t]{4}{*}{$\mathrm{Fe} \mathrm{K} \alpha$} & $\mathrm{LE}(\mathrm{keV})$ & $6.44_{-0.04}^{+0.03}$ & $6.38_{-0.04}^{+0.04}$ & $6.41_{-0.06}^{+0.03}$ & $6.39_{-0.10}^{+0.07}$ & $6.29_{-0.14}^{+0.08}$ & $6.28_{-0.11}^{+0.07}$ & $6.29_{-0.09}^{+0.05}$ & $6.19_{-0.10}^{+0.05}$ \\
\hline & $\mathrm{EW}(\mathrm{eV})$ & $206_{-18}^{+4}$ & $114_{-15}^{+11}$ & $126_{-21}^{+3}$ & $117_{-10}^{+14}$ & $106_{-6}^{+1}$ & $<95$ & $155_{-16}^{+12}$ & $108_{-22}^{+15}$ \\
\hline & FWHM $\left(\mathrm{km} \mathrm{s}^{-1}\right)$ & $2329^{*}$ & $8695_{-1243}^{+924}$ & $2108^{*}$ & $4796_{-982}^{+863}$ & $2337^{*}$ & $4461_{-845}^{+1045}$ & $6025_{-1194}^{+946}$ & $6613_{-1223}^{+1275}$ \\
\hline & Norm $\left(10^{-5}\right)$ & $6.83_{-0.22}^{+0.15}$ & $11.74_{-1.22}^{+1.10}$ & $3.47_{-0.10}^{+0.14}$ & $2.56_{-0.13}^{+0.18}$ & $1.91_{-0.09}^{+0.07}$ & $0.93_{-0.10}^{+0.04}$ & $2.14_{-0.13}^{+0.10}$ & $2.43_{-0.08}^{+0.10}$ \\
\hline \multirow[t]{4}{*}{ Fe XXVI } & $\mathrm{LE}(\mathrm{keV})$ & - & $6.87_{-0.04}^{+0.04}$ & $6.89_{-0.03}^{+0.04}$ & $7.04_{-0.04}^{+0.05}$ & $6.93_{-0.03}^{+0.04}$ & - & - & - \\
\hline & $\mathrm{EW}(\mathrm{eV})$ & - & $<37$ & $<49$ & $<92$ & $<42$ & - & - & - \\
\hline & FWHM $\left(\mathrm{km} \mathrm{s}^{-1}\right)$ & - & $<4452$ & $<4049$ & $<4943$ & $<3913$ & - & - & - \\
\hline & Norm $\left(10^{-6}\right)$ & - & $21.09_{-0.94}^{+1.02}$ & $7.88_{-0.22}^{+0.13}$ & $8.95_{-0.94}^{+1.06}$ & $7.30_{-0.73}^{+0.31}$ & - & - & - \\
\hline \multicolumn{2}{|c|}{$k T_{\mathrm{bb} 1}(\mathrm{eV})$} & $116_{-8}^{+7}$ & $112_{-8}^{+6}$ & $117_{-7}^{+11}$ & $115_{-9}^{+5}$ & $108_{-12}^{+8}$ & $114_{-6}^{+9}$ & $117_{-14}^{+12}$ & $122_{-10}^{+17}$ \\
\hline \multicolumn{2}{|c|}{$N_{\text {bb1 }}\left(10^{-5}\right)$} & $0.49_{-0.06}^{+0.03}$ & $28.6_{-3.42}^{+2.29}$ & $8.30_{-0.65}^{+1.09}$ & $4.35_{-0.96}^{+0.76}$ & $5.33_{-1.04}^{+0.51}$ & $0.98_{-0.19}^{+0.24}$ & $4.71_{-1.13}^{+0.72}$ & $2.65_{-0.56}^{+0.80}$ \\
\hline \multicolumn{2}{|l|}{$\chi^{2} / \mathrm{dof}$} & $1073 / 998$ & $2744 / 2559$ & $2224 / 2127$ & $1608 / 1646$ & $693 / 654$ & $835 / 834$ & $535 / 542$ & $576 / 567$ \\
\hline \multicolumn{2}{|c|}{$N_{\mathrm{H}, 1}\left(10^{21} \mathrm{~cm}^{-2}\right)$} & $3.46_{-0.05}^{+0.06}$ & $0.78_{-0.21}^{+0.10}$ & $0.96_{-0.16}^{+0.17}$ & $1.22_{-0.15}^{+0.13}$ & $1.32_{-0.16}^{+0.12}$ & $1.19_{-0.09}^{+0.10}$ & $1.26_{-0.18}^{+0.12}$ & $1.27_{-0.19}^{+0.15}$ \\
\hline \multicolumn{2}{|l|}{$\log \xi_{1}$} & $-3^{\dagger}$ & $1.66_{-0.08}^{+0.10}$ & $1.85_{-0.11}^{+0.06}$ & $1.32_{-0.10}^{+0.07}$ & $1.05_{-0.06}^{+0.04}$ & $0.26_{-0.07}^{+0.05}$ & $0.15_{-0.07}^{+0.12}$ & $0.21_{-0.05}^{+0.04}$ \\
\hline \multicolumn{2}{|c|}{ Cov Frac1 } & $0.20_{-0.05}^{+0.07}$ & $0.46_{-0.03}^{+0.03}$ & $0.32_{-0.04}^{+0.07}$ & $0.34_{-0.14}^{+0.05}$ & $<0.12$ & $0.22_{-0.16}^{+0.11}$ & $<0.15$ & $<0.1$ \\
\hline \multicolumn{2}{|c|}{$N_{\mathrm{H}, 2}\left(10^{21} \mathrm{~cm}^{-2}\right)$} & - & $4.25_{-0.23}^{+0.32}$ & $4.50_{-0.43}^{+0.64}$ & $0.82_{-0.19}^{+0.27}$ & - & - & - & - \\
\hline \multicolumn{2}{|l|}{$\log \xi_{2}$} & - & $4.63_{-0.09}^{+0.45}$ & $3.32_{-0.10}^{+0.33}$ & $3.11_{-0.16}^{+0.23}$ & - & - & - & - \\
\hline \multicolumn{2}{|c|}{ Cov Frac 2} & - & $>0.75$ & $0.59_{-0.25}^{+0.22}$ & $0.56_{-0.45}^{+0.23}$ & - & - & - & - \\
\hline \multicolumn{2}{|l|}{$\Gamma$} & $1.74_{-0.07}^{+0.05}$ & $1.88_{-0.06}^{+0.04}$ & $1.75_{-0.03}^{+0.03}$ & $1.72_{-0.03}^{+0.02}$ & $1.71_{-0.05}^{+0.03}$ & $1.69_{-0.04}^{+0.04}$ & $1.68_{-0.05}^{+0.05}$ & $1.69_{-0.05}^{+0.04}$ \\
\hline \multicolumn{2}{|c|}{$k T_{\mathrm{e}}(\mathrm{keV})$} & $101.8_{-4.3}^{+4.9}$ & $60.8_{-6.7}^{+5.5}$ & $85.7_{-5.9}^{+7.4}$ & $92.2_{-9.4}^{+8.4}$ & $75.2_{-7.6}^{+8.3}$ & $104.2_{-6.9}^{+5.6}$ & $105.8_{-5.5}^{+6.9}$ & $\begin{array}{r}96.7_{-8.2}^{+7.5}\end{array}$ \\
\hline \multicolumn{2}{|l|}{$\tau$} & $1.27_{-0.11}^{+0.17}$ & $1.60_{-0.18}^{+0.33}$ & $1.43_{-0.15}^{+0.16}$ & $1.41_{-0.12}^{+0.17}$ & $1.66_{-0.17}^{+0.24}$ & $1.30_{-0.12}^{+0.14}$ & $1.34_{-0.13}^{+0.15}$ & $1.41_{-0.13}^{+0.19}$ \\
\hline
\end{tabular}

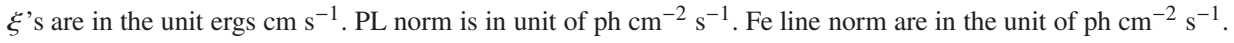

$\tau$ 's are calculated using Eqn. 1.

$\dagger$ pegged at the lowest value.

* Gaussian fitted with fixed line width, $\sigma=0.05 \mathrm{keV}$. 

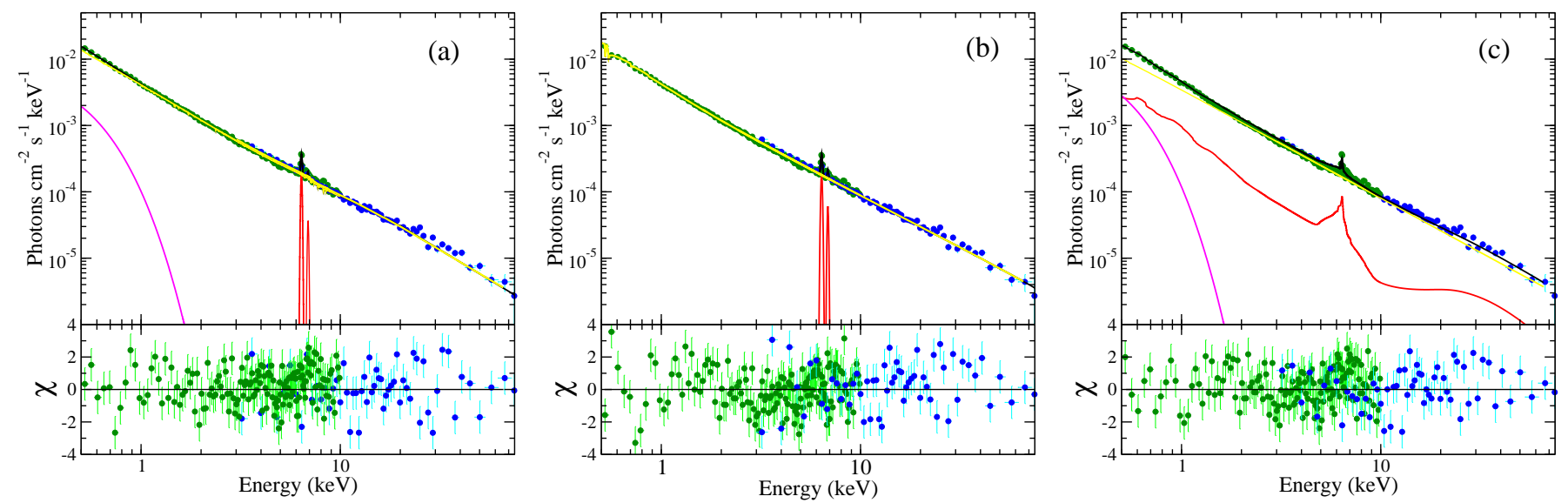

Figure 6. Best-fit unfolded spectra using Model-1, Model-3 and Model-4 are shown in the left, middle, and right panel, respectively, for O2. The corresponding residuals are shown in the bottom of each panel. Left panel: the black, yellow, magenta and red lines represent the total, primary emission, soft excess and Fe line emission, respectively. Middle panel: the black, yellow, and red lines represent the total, the AGN emission and Fe line emission, respectively. Right panel: the black, yellow, magenta and red lines represent the total, primary emission, soft excess and reflection component, respectively.

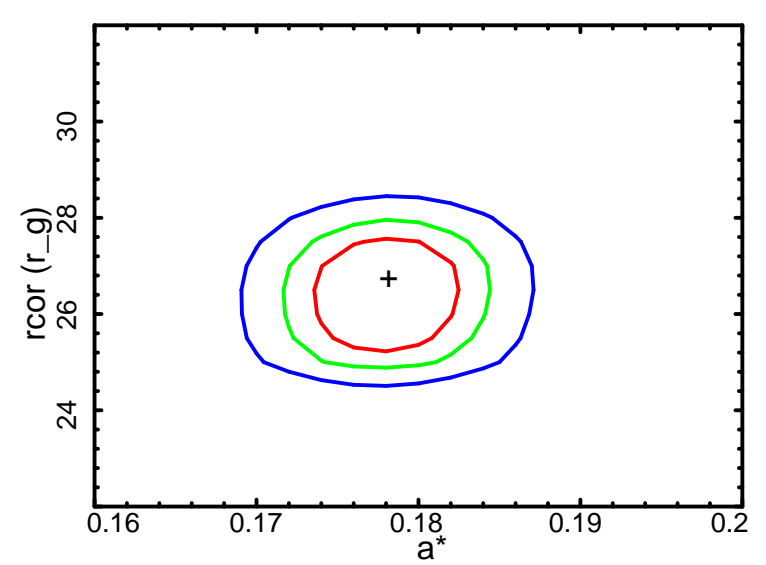

Figure 7. 2D contour plot between $R_{\text {cor }}$ and $a^{*}$ for Model-3 during the observation $\mathrm{O} 2$.

nearly constant photon index and a variable temperature would imply a variation in the density of the Comptonizing cloud. This would suggest that the optical depth was varying during the observations in the range of $\sim 1.27-1.66$. The results obtained from our spectral fitting with this model are listed in Table 4.

\subsubsection{Model 3: OPTXAGNF}

The X-ray spectra of AGN typically show a soft excess below $2 \mathrm{keV}$ (Arnaud et al. 1985; Singh et al. 1985). Although the soft excess in AGNs was first detected in the 1980s, its origin is still very debated. In models $1 \& 2$, we fitted the soft excess component with a phenomenological blackbody model. To shed light on the origin of the soft excess in this source, we used a more physical model, OPTXAGNF (Done et al. 2012).

The OPTXAGNF model (hereafter Model-3) computes the soft excess and the primary emission self-consistently. In this model, total emission is determined by the mass accretion rate and by the $\mathrm{BH}$ mass. The disc emission emerges as a colour temperature corrected blackbody emission at radii $R_{\text {out }}>r>R_{\text {cor }}$, where $R_{\text {out }}$ and $R_{\text {cor }}$ are the outer edge of the disc and the corona, respectively. At $r<R_{\text {cor }}$, the disc emission emerges as the Comptonized emission from a warm and optically-thick medium, rather than thermal emission. The hot and optically-thin corona is located around the disc and produces the high energy power-law continuum. The total Comptonized emission is divided between the cold and hot corona, and the fraction of the hot-Comptonized emission $\left(f_{\mathrm{PL}}\right)$ can be found from the model fitting. The temperature of the cold corona $\left(k T_{\mathrm{S}}\right)$, temperature of the seed photon, and the optical depth of the cold corona $(\tau)$ at $r=R_{\text {cor }}$ determine the energy of the up-scattered soft excess emission. The power-law continuum is approximated as the NTHCOMP model, with the seed photon temperature fixed at the disc temperature at $r=R_{\text {cor }}$, and the electron temperature fixed at $100 \mathrm{keV}$.

When using the OPTXAGNF model, we included two Gaussian components to incorporate the $\mathrm{Fe}$ emission lines. The model reads in $\mathrm{XSPEC}$ as,

$\mathrm{TB} \times \operatorname{zxipcf} 1 \times \operatorname{zxipcf} 2 \times($ OPTXAGNF $+z G a+z G a)$.

While fitting the data with this model, we kept the $\mathrm{BH}$ mass frozen at $M_{\mathrm{BH}}=8.3 \times 10^{6} M_{\odot}$ (Woo \& Urry 2002). As recommended, we fixed normalization to unity during our analysis. Initially, we started our analysis with one absorption component. However, an absorption feature was seen during $\mathrm{O} 1, \mathrm{O} 2$ and $\mathrm{O} 3$. Thus, we added second absorption component in the spectra of $\mathrm{O} 1, \mathrm{O} 2$ and $\mathrm{O} 3$. Adding second zxipcf component significantly improved the fit with $\Delta \chi^{2}=88,76$, 65 for 3 dof, during the observation $\mathrm{O} 1, \mathrm{O} 2$ and $\mathrm{O} 3$, respectively. Overall, this model provided a good fit for all the observations. A clear variation in the Eddington ratio and size of the corona were observed in the different observations. In the rising phase of the 2018 outburst (observation O1), a high Eddington ratio $\left(L / L_{\text {Edd }} \sim 0.23\right)$ and a large corona $\left(R_{\text {cor }}=43 \pm 3 R_{\mathrm{g}}\right)$ were observed. These values were found to be higher than the pre-outburst observation $\left(\mathrm{X} 1 ; L / L_{\mathrm{Edd}} \sim 0.04\right.$; $R_{\text {cor }}=12_{-1}^{+2} R_{\mathrm{g}}$ ). In later observations, both Eddington ratio and size of the X-ray corona decreased to $L / L_{\text {Edd }} \sim 0.06-0.07$ and $R_{\text {cor }} \sim 15 \pm 2-26 \pm 2 R_{g}$, respectively. In the observation O1, the electron temperature of the optically thick Comptonizing region was observed to be $k T_{\mathrm{S}} \sim 1.4 \pm 0.1 \mathrm{keV}$, along with optical depth $\tau \sim 4.6 \pm 1$. The temperature of the optically-thick medium decreased to $k T_{\mathrm{S}} \sim 0.5-0.6 \mathrm{keV}$ in the later observations $(\mathrm{O} 3-\mathrm{O} 7)$. However, the optical depth did not change much and varied in the range of $\tau \sim 4-5$. A significant fraction of the Comptonized emission was 
emitted from the optically thin corona with $f_{\mathrm{PL}}>0.79$ during all the observations. We allowed the spin of the BH to vary. The best-fitted spin parameter fluctuated in the range of $a^{*} \sim 0.18_{-0.02}^{+0.01}-0.21_{-0.02}^{+0.03}$, suggesting that the SMBH is spinning slowly. The variation of the column density $\left(N_{\mathrm{H}}\right)$ and of the photon indices $(\Gamma)$ was similar to that observed using Model-1. All the parameters obtained from our spectral analysis using Model-3 are presented in Table 5. In Fig. 6b, we show the unfolded spectrum fitted with Model-3 for observation O2. In Fig. 7, we display the contour plot of $R_{\text {cor }}$ and $a^{*}$, which shows that there is no strong degeneracy between those two parameters.

\subsubsection{Model-4 : RELXILL}

Reprocessed X-ray radiation is a feature often observed in AGN spectra. This reflection component typically consists of a reflection hump at $\sim 15-40 \mathrm{keV}$ and fluorescent iron lines. In Model $1-3$ we did not include a reprocessed X-ray radiation, hence, we add a relativistic reflection component RELXILL (García et al. 2014; Dauser et al. 2014, 2016) to our baseline model.

In this model, the strength of reflection is measured from the relative reflection fraction $\left(R_{\text {refl }}\right)$, which is defined as the ratio between the observed Comptonized emission and the radiation reprocessed by the disc. RELXILL assumes a broken power-law emission profile $\left(E(r) \approx r^{-q}\right)$, where $r$ is the distance from the SMBH, $E(r)$ is the emissivity, and $q$ is the emissivity index. At a larger disc radii, in a non-relativistic domain, the emissivity profile has a form of $E(r) \sim r^{-3}$. However, in the relativistic domain, the emissivity profile is steeper. The break radius $\left(R_{\mathrm{br}}\right)$ separates the relativistic and non-relativistic domains. In our analysis, we fixed $q_{2}=3$ for emission at $r>R_{\mathrm{br}}$.

In our spectral analysis, we used RELXILL along with the absorbed power-law continuum. We only considered the reflection component from RELXILL model by setting $R_{\text {refl }}$ to a negative value. The model (hereafter Model-4) read in XSPEC as,

TB $\times$ zxipcf1 $\times$ zxipcf2 $\times(z P L 1+$ RELXILL + bbody).

While fitting the data with this model, we tied the photon indices of the RELXILL component to that of the power-law model. Although, we started our analysis with one absorption component, we required two absorption component during $\mathrm{O} 1, \mathrm{O} 2$ and $\mathrm{O} 3$. The second absorption component improved the fit with $\Delta \chi^{2}=82,68$ and 59 for 3 dof, during O1, O2 and O3 respectively. Throughout the observations, we obtained a fairly unchanged value of the iron abundances with $A_{\mathrm{Fe}} \sim 3.7-4.2 A_{\odot}$. Disc ionization was also constant during our observation period with $\xi \sim 10^{1.9-2.2} \mathrm{erg} \mathrm{cm} \mathrm{s}^{-1}$. The emissivity profile was quite stable with $q_{2} \sim 4-5$, although $R_{\mathrm{br}}$ was found to change. This parameter reached its maximum during the observation $\mathrm{O} 1\left(R_{\mathrm{br}}=42_{-2}^{+3} R_{g}\right)$. In the later observations, it decreased and varied in the range of $R_{\mathrm{br}} \sim 16-26 R_{g}$. The inner edge of the disc varied in the range of $R_{\text {in }} \sim 4-7 R_{g}$. The best-fit inclination angle of the AGN was obtained in the range of $10.3_{-6.1}^{+4.9}$ $17.7_{-5.2}^{+2.9}$. The spin of the BH in NGC 1566 was observed to be low, with the best-fitted spin parameters found to be in the range of $0.15_{-0.03}^{+0.03}-0.21_{-0.02}^{+0.04}$, which is consistent with what we found from the OPTXAGNF model. In all the observations, the reflection component was found to be relatively weak with reflection fraction varied in the range of, $R_{\text {refl }} \sim 0.10-0.18$. The RELXILL model fitted spectral analysis results are given in Table 6 . We show the unfolded spectrum fitted with Model-4 for observation $\mathrm{O} 2$ in Figure 6. In Fig. 8, we show the contour plot of $R_{\text {in }}$ and $a^{*}$ for the observation $\mathrm{O} 2$.

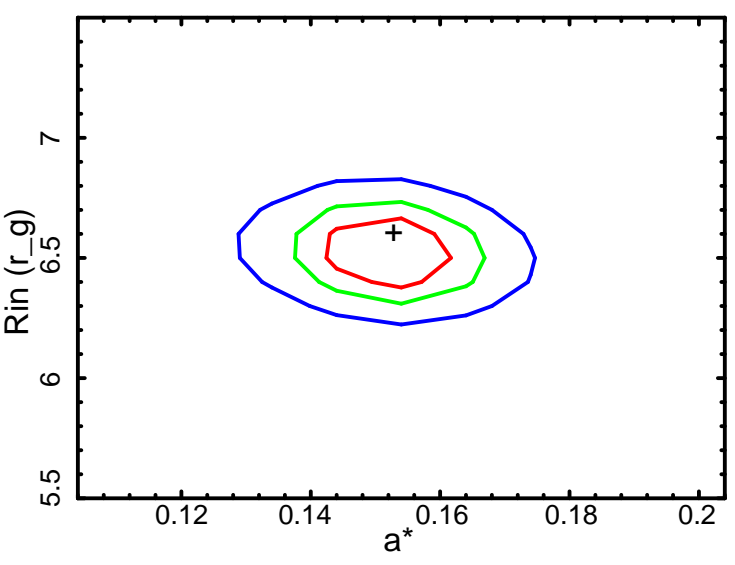

Figure 8. 2D contour plot between $R_{\text {in }}$ and $a^{*}$ for Model-4 during the observation $\mathrm{O} 2$

\section{DISCUSSION}

We studied NGC 1566 during and after the 2018 outburst event using data from XMM-Newton, Swift and NUSTAR in the $0.5-$ $70 \mathrm{keV}$ energy band. From a detailed spectral and timing analysis, we explored the nuclear properties of the AGN.

\subsection{Black Hole Properties}

NGC 1566 hosts a supermassive black hole of mass $M_{\mathrm{BH}} \approx 8.3 \times 10^{6}$ $M_{\odot}$ (Woo \& Urry 2002). We kept the mass of the BH frozen during our spectral analysis with Model-3. Fitting the spectra with Model-3 and Model-4, we estimated the spin parameter $\left(a^{*}\right)$ to be in the range $0.18_{-0.02}^{+0.01}-0.21_{-0.02}^{+0.03}$ and $0.15_{-0.3}^{+0.2}-0.21_{-0.02}^{+0.04}$, respectively. Both models favour a low spinning $\mathrm{BH}$ with the spin parameter $a^{*} \sim 0.2$, which is consistent with the findings of Parker et al. (2019).

The inclination angle was a free parameter in Model-4, and it was estimated to be in the range of $10.3_{-6.1}^{+4.9}{ }^{\circ} 17.7_{-5.2}^{+2.9}$. Parker et al. (2019) also found a consistent inclination angle $\left(\theta_{\text {incl }}<11^{\circ}\right)$.

\subsection{Corona Properties}

The X-ray emitting corona is generally located very close to the central BH (Fabian et al. 2015). This corona is characterized by the photon index $(\Gamma)$, temperature $\left(k T_{\mathrm{e}}\right)$ and optical depth $(\tau)$ of the Comptonizing plasma. While using a simple power-law model gives us information only about the photon index, the NTHCOMP model can provide us with information on the electron temperature of the Compton cloud $\left(k T_{\mathrm{e}}\right)$, while the optical depth $(\tau)$ is calculated from Equation 7 . We found that the photon index varied within a narrow range of $\Gamma \sim 1.7-1.8$, and can be considered as constant within the uncertainties. To constrain the photon index with more accuracy, we fitted the source spectra from NUSTAR observations in $3-70 \mathrm{keV}$ and $10-70 \mathrm{keV}$ energy ranges to approximate only the power-law part. We found similar results as from the simultaneous fitting of the XMM-Newton and NUSTAR data in $0.5-70 \mathrm{keV}$ range. From the spectral analysis with Model-3, we estimated the Compton cloud radius to be $12-43 R_{\mathrm{g}}$.

We calculated the intrinsic luminosity $\left(L_{0.1-100}\right)$ of the AGN from Model-1. The intrinsic luminosity was observed to be low during the pre-outburst observation, X1, with $L_{0.1-100} \sim(4.3 \pm$ $0.1) \times 10^{41} \mathrm{erg} \mathrm{s}^{-1}$. During O1, was observed to be maximum with 
Table 5. Best-fit parameters obtained from the spectral fitting of the source spectra with Model-3 (OPTXAGNF).

\begin{tabular}{|c|c|c|c|c|c|c|c|c|}
\hline & $\mathrm{X} 1$ & $\mathrm{O} 1$ & $\mathrm{O} 2$ & $\mathrm{O} 3$ & $\mathrm{O} 4$ & O5 & O6 & O7 \\
\hline$N_{\mathrm{H}, 1}\left(10^{21} \mathrm{~cm}^{-2}\right)$ & $3.49_{-0.05}^{+0.05}$ & $\begin{array}{l}0.80_{-0.16}^{+0.08} \\
\end{array}$ & $0.94_{-0.15}^{+0.15}$ & $\begin{array}{r}1.20_{-0.11}^{+0.15} \\
\end{array}$ & $1.34_{-0.12}^{+0.10}$ & $1.17_{-0.11}^{+0.08}$ & $1.25_{-0.14}^{+0.08}$ & $\begin{array}{l}1.29_{-0.22}^{+0.15} \\
\end{array}$ \\
\hline $\log \xi_{1}$ & $-3^{\dagger}$ & $1.67_{-0.06}^{+0.07}$ & $1.82_{-0.06}^{+0.03}$ & $1.30_{-0.09}^{+0.07}$ & $1.08_{-0.10}^{+0.07}$ & $0.23_{-0.10}^{+0.07}$ & $0.18_{-0.04}^{+0.06}$ & $0.20_{-0.04}^{+0.03}$ \\
\hline Cov Frac2 & $0.18_{-0.06}^{+0.08}$ & $0.45_{-0.04}^{+0.03}$ & $0.34_{-0.06}^{+0.09}$ & $0.35_{-0.11}^{+0.05}$ & $<0.12$ & $0.21_{-0.17}^{+0.12}$ & $<0.14$ & $<0.15$ \\
\hline$N_{\mathrm{H}, 2}\left(10^{21} \mathrm{~cm}^{-2}\right)$ & - & $4.28_{-0.38}^{+0.45}$ & $4.56_{-0.46}^{+0.51}$ & $0.89_{-0.16}^{+0.21}$ & - & - & - & - \\
\hline $\log \xi_{2}$ & - & $4.52_{-0.22}^{+0.39}$ & $3.15_{-0.18}^{+0.21}$ & $2.93_{-0.19}^{+0.32}$ & - & - & - & - \\
\hline Cov Frac2 & - & $>0.79$ & $0.54_{-0.17}^{+0.22}$ & $0.51_{-0.21}^{+0.28}$ & - & - & - & - \\
\hline$L / L_{\mathrm{Edd}}$ & $-1.98_{-0.04}^{+0.03}$ & $-0.64_{-0.02}^{+0.02}$ & $-0.78_{-0.03}^{+0.03}$ & $-1.16_{-0.04}^{+0.03}$ & $-0.81_{-0.03}^{+0.02}$ & $-0.97_{-0.05}^{+0.04}$ & $-1.20_{-0.03}^{+0.03}$ & $-1.17_{-0.01}^{+0.02}$ \\
\hline$a^{*}$ & $0.19_{-0.01}^{+0.01}$ & $0.18_{-0.02}^{+0.01}$ & $0.18_{-0.01}^{+0.01}$ & $0.21_{-0.02}^{+0.01}$ & $0.20_{-0.03}^{+0.02}$ & $0.19_{-0.02}^{+0.03}$ & $0.21_{-0.02}^{+0.03}$ & $0.19_{-0.01}^{+0.01}$ \\
\hline$R_{\text {cor }}\left(R_{\mathrm{g}}\right)$ & $12_{-1}^{+2}$ & $43_{-2}^{+3}$ & $26_{-2}^{+2}$ & $15_{-2}^{+1}$ & $22_{-2}^{+2}$ & $20_{-3}^{+2}$ & $17_{-3}^{+2}$ & $18_{-1}^{+1}$ \\
\hline$k T_{\mathrm{S}}(\mathrm{keV})$ & $0.32_{-0.05}^{+0.04}$ & $1.39_{-0.15}^{+0.10}$ & $0.90_{-0.11}^{+0.07}$ & $0.65_{-0.10}^{+0.05}$ & $0.61_{-0.06}^{+0.08}$ & $0.56_{-0.08}^{+0.07}$ & $0.59_{-0.03}^{+0.07}$ & $0.54_{-0.03}^{+0.04}$ \\
\hline$\tau$ & $4.16_{-0.21}^{+0.28}$ & $4.56_{-0.14}^{+0.12}$ & $4.30_{-0.20}^{+0.27}$ & $4.33_{-0.21}^{+0.18}$ & $5.23_{-0.19}^{+0.30}$ & $4.92_{-0.39}^{+0.34}$ & $4.29_{-0.45}^{+0.39}$ & $3.54_{-0.46}^{+0.34}$ \\
\hline$\Gamma$ & $\begin{array}{r}1.77_{-0.03}^{+0.03}\end{array}$ & $1.74_{-0.02}^{+0.02}$ & $1.66_{-0.05}^{+0.04}$ & $1.73_{-0.04}^{+0.02}$ & $1.72_{-0.04}^{+0.02}$ & $\begin{array}{r}1.70_{-0.02}^{+0.03}\end{array}$ & $1.72_{-0.02}^{+0.02}$ & $1.66_{-0.03}^{+0.03}$ \\
\hline$f_{\mathrm{PL}}$ & $0.84_{-0.02}^{+0.01}$ & $0.79_{-0.02}^{+0.01}$ & $0.81_{-0.02}^{+0.03}$ & $0.84_{-0.03}^{+0.03}$ & $0.88_{-0.03}^{+0.03}$ & $0.83_{-0.04}^{+0.03}$ & $0.88_{-0.04}^{+0.03}$ & $0.94_{-0.03}^{+0.03}$ \\
\hline$\chi^{2} / \mathrm{dof}$ & $1070 / 995$ & $2784 / 2551$ & $2311 / 2120$ & $1677 / 1643$ & $706 / 649$ & $827 / 831$ & $526 / 535$ & $571 / 560$ \\
\hline
\end{tabular}

$\xi$ 's are in the unit ergs $\mathrm{cm} \mathrm{s}^{-1}$. $\dagger$ pegged at the lowest value.

$L_{0.1-100} \sim(1.39 \pm 0.01) \times 10^{44} \mathrm{erg} \mathrm{s}^{-1}$. Later, the intrinsic luminosity decreased and varied in the range of $2.1-4.3 \times 10^{43}$ $\mathrm{erg} \mathrm{s}^{-1}$. We also computed the luminosity for the primary emission $\left(L_{\text {nuc }}\right)$ and soft excess $\left(L_{\text {soft }}\right)$ from the individual components while analyzing the spectra with Model-1. We calculated the bolometric luminosity $\left(L_{\text {bol }}\right)$ using the $2-10 \mathrm{keV}$ bolometric correction, $\kappa_{\text {bol,2-10 } \mathrm{keV}}=20$ (Vasudevan $\&$ Fabian 2009). The Eddington ratio $\left(\lambda_{\mathrm{Edd}}=L_{\mathrm{bol}} / L_{\mathrm{Edd}}\right)$, assuming a $\mathrm{BH}$ of mass of $8.3 \times 10^{6}$ $M_{\odot}\left(\right.$ Woo \& Urry 2002), was estimated to be $\lambda_{\text {Edd }} \sim 0.003-0.066$ in different epoch which is consistent with other nearby Seyfert-1 galaxies (Wu \& Liu 2004; Sikora et al. 2007; Koss et al. 2017).

In the pre-outburst observation in November 2015 (X1 : see Table 7), we obtained a bolometric luminosity of $L_{\text {bol }}=(0.9 \pm 0.1) \times$ $10^{42} \mathrm{erg} \mathrm{s}^{-1}$, with corona size $R_{\text {cor }}=12 \pm 3 R_{\mathrm{g}}$ and hot electron plasma temperature $k T_{\mathrm{e}}=102 \pm 5 \mathrm{keV}$. In the observation during the outburst in June 2018 (O1), the luminosity of the AGN increased by a factor of about $\sim 25$, compared to the November 2015 observation (X1). During this observation, the corona was large $\left(R_{\text {cor }}=43 \pm 3 R_{\mathrm{g}}\right)$ with hot electron plasma temperature $k T_{\mathrm{e}}=61 \pm 7 \mathrm{keV}$ and the observed spectrum was harder. As the outburst progressed, the bolometric luminosity and the corona size decreased. As $R_{\text {cor }}$ decreased, the electron plasma temperature increased. Overall, $k T_{\mathrm{e}}$ varied in a range of $\sim 61-106 \mathrm{keV}$ during the observations. In general, the plasma temperature is observed in a wide range, with a median at $k T_{\mathrm{e}} \sim 105 \pm 18 \mathrm{keV}$ (Ricci et al. 2018). Thus, the plasma temperature is consistent with other AGNs. During these observations, the optical depth of the Compton cloud varied within $\sim 1.2-1$.7. Interestingly, the photon index $(\Gamma)$ was almost constant, although some of the properties of the corona evolved with time. This appears to imply that both the optical depth and the hot electron temperature changed in such a way that the spectral shape remained the same.

We found several correlations and anti-correlations between the spectral parameters and show them in Fig. 9. We fitted the data points with linear regression method using $y=m x+c$. The fitted value of the slope (m) and intercept (c) are mentioned in each panel of Fig. 9. We found that the nuclear luminosity ( $\left.L_{\text {nuc }}\right)$ and the soft excess luminosity $\left(L_{\text {soft }}\right)$ are strongly correlated with the Eddington ratio with the Pearson correlation indices of 0.84 and 0.85 , respectively. We also found that the bolometric luminosity $\left(L_{\mathrm{bol}}\right)$ and the Compton cloud temperature $\left(k T_{\mathrm{e}}\right)$ are anti-correlated $\left(\rho_{\mathrm{S}}=-0.85\right)$. The electron temperature is found to be anti-correlated with the Eddington ratio $\left(\rho_{\mathrm{S}}=-0.85\right)$, while the size of the Compton corona and the luminosity are positively correlated $\left(\rho_{\mathrm{S}}=0.93\right)$. We also observed that the electron temperature is anti-correlated with the size of the Compton cloud $\left(\rho_{\mathrm{S}}=-0.84\right)$. The above correlations can be explained thinking that, as the accretion rate increased, the energy radiation increased, thereby, increasing the luminosity. An increase in the mass accretion rate makes the cooling more efficient, leading to a decrease in the electron temperature of the Comptonizing region (Haardt \& Maraschi 1991; Done et al. 2007).

\subsection{Reflection}

The hard X-ray photons from the corona are reflected from cold material in the accretion disc, BLR and torus, producing a reflection hump and a Fe-K emission line (George \& Fabian 1991; Matt et al. 1991). When fitted with a simple power-law model, NGC 1566 showed the presence of $\mathrm{Fe} \mathrm{K} \alpha$ emission line along with a weak reflection hump at $\sim 15-40 \mathrm{keV}$ energy range (see Fig 6). Thus, we fitted the spectra 
Table 6. Best-fit parameters obtained from the spectral fitting of the source spectra with Model-4 (RELXILL).

\begin{tabular}{|c|c|c|c|c|c|c|c|c|}
\hline & $\mathrm{X} 1$ & $\mathrm{O} 1$ & $\mathrm{O} 2$ & $\mathrm{O} 3$ & $\mathrm{O} 4$ & O5 & O6 & O7 \\
\hline$N_{\mathrm{H}, 1}\left(10^{21} \mathrm{~cm}^{-2}\right)$ & $3.51_{-0.04}^{+0.03}$ & $0.77_{-0.11}^{+0.06}$ & $0.95_{-0.14}^{+0.11}$ & $1.18_{-0.10}^{+0.12}$ & $1.28_{-0.10}^{+0.08}$ & $1.16_{-0.06}^{+0.07}$ & $1.28_{-0.08}^{+0.10}$ & $1.31_{-0.18}^{+0.12}$ \\
\hline $\log \xi_{1}$ & $-3^{\dagger}$ & $1.63_{-0.04}^{+0.05}$ & $1.85_{-0.04}^{+0.05}$ & $1.31_{-0.08}^{+0.07}$ & $1.05_{-0.08}^{+0.05}$ & $0.21_{-0.06}^{+0.05}$ & $0.19_{-0.03}^{+0.06}$ & $0.24_{-0.03}^{+0.04}$ \\
\hline Cov Frac2 & $0.17_{-0.06}^{+0.05}$ & $0.44_{-0.06}^{+0.04}$ & $0.36_{-0.08}^{+0.05}$ & $0.33_{-0.12}^{+0.06}$ & $<0.14$ & $<0.18$ & $<0.16$ & $<0.13$ \\
\hline$N_{\mathrm{H}, 2}\left(10^{21} \mathrm{~cm}^{-2}\right)$ & - & $4.36_{-0.47}^{+0.34}$ & $4.58_{-0.42}^{+0.37}$ & $0.95_{-0.26}^{+0.19}$ & - & - & - & - \\
\hline $\log \xi_{2}$ & - & $4.58_{-0.26}^{+0.41}$ & $3.10_{-0.24}^{+0.19}$ & $2.96_{-0.29}^{+0.26}$ & - & - & - & - \\
\hline Cov Frac2 & - & $>0.74$ & $0.52_{-0.23}^{+0.18}$ & $0.53_{-0.25}^{+0.31}$ & - & - & - & - \\
\hline$\Gamma$ & $1.77_{-0.04}^{+0.03}$ & $1.76_{-0.03}^{+0.05}$ & $1.68_{-0.02}^{+0.04}$ & $1.73_{-0.03}^{+0.03}$ & $1.65_{-0.03}^{+0.04}$ & $1.67_{-0.03}^{+0.05}$ & $1.71_{-0.03}^{+0.02}$ & $1.68_{-0.04}^{+0.03}$ \\
\hline$N_{\mathrm{PL}}\left(10^{-3} \mathrm{ph} \mathrm{cm}^{-2} \mathrm{~s}^{-1}\right)$ & $1.48_{-0.14}^{+0.10}$ & $22.35_{-0.93}^{+1.02}$ & $\begin{array}{r}6.15_{-0.68}^{+0.45} \\
\end{array}$ & $2.54_{-0.47}^{+0.32}$ & $4.41_{-0.57}^{+0.41}$ & $2.82_{-0.32}^{+0.26}$ & $2.72_{-0.22}^{+0.15}$ & $2.55_{-0.28}^{+0.21}$ \\
\hline$A_{\mathrm{Fe}}\left(A_{\odot}\right)$ & $3.68_{-0.28}^{+0.22}$ & $3.89_{-0.35}^{+0.26}$ & $4.06_{-0.22}^{+0.32}$ & $4.15_{-0.38}^{+0.34}$ & $3.77_{-0.38}^{+0.27}$ & $4.12_{-0.37}^{+0.27}$ & $3.95_{-0.42}^{+0.38}$ & $4.18_{-0.39}^{+0.27}$ \\
\hline $\log (\xi)$ & $1.96_{-0.02}^{+0.03}$ & $2.09_{-0.04}^{+0.05}$ & $2.18_{-0.02}^{+0.03}$ & $2.11_{-0.02}^{+0.02}$ & $2.07_{-0.03}^{+0.02}$ & $2.18_{-0.03}^{+0.04}$ & $2.13_{-0.03}^{+0.02}$ & $1.98_{-0.02}^{+0.02}$ \\
\hline$\theta_{\text {incl }}$ (degree) & $13.8_{-4.1}^{+2.7}$ & $11.8_{-5.5}^{+4.1}$ & $16.2_{-4.7}^{+2.8}$ & $14.3_{-3.9}^{+4.4}$ & $\begin{array}{r}10.3_{-6.1}^{+4.9} \\
-\end{array}$ & $13.2_{4.7}^{+2.5}$ & $\begin{array}{r}17.7_{-5.2}^{+2.9} \\
\end{array}$ & $12.1_{-5.8}^{+4.5}$ \\
\hline$R_{\text {refl }}$ & $0.11_{-0.02}^{+0.02}$ & $0.16_{-0.04}^{+0.03}$ & $0.15_{-0.02}^{+0.03}$ & $0.13_{-0.02}^{+0.02}$ & $0.18_{-0.03}^{+0.02}$ & $0.16_{-0.02}^{+0.03}$ & $0.10_{-0.02}^{+0.03}$ & $0.12_{-0.02}^{+0.03}$ \\
\hline$q_{2}$ & $3.66_{-0.41}^{+0.17}$ & $4.69_{-0.35}^{+0.68}$ & $4.41_{-0.37}^{+0.25}$ & $5.35_{-0.29}^{+0.45}$ & $5.31_{-0.41}^{+0.29}$ & $4.81_{-0.63}^{+0.42}$ & $4.56_{-0.89}^{+0.72}$ & $4.94_{-0.76}^{+0.60}$ \\
\hline$R_{\mathrm{br}}\left(R_{g}\right)$ & $12_{-2}^{+3}$ & $42_{-2}^{+3}$ & $26_{-3}^{+2}$ & $17_{-1}^{+3}$ & $18_{-3}^{+2}$ & $21_{-3}^{+2}$ & $21_{-1}^{+2}$ & $16_{-3}^{+2}$ \\
\hline$a^{*}$ & $0.15_{-0.03}^{+0.02}$ & $0.16_{-0.03}^{+0.03}$ & $0.15_{-0.03}^{+0.03}$ & $0.17_{-0.03}^{+0.02}$ & $0.21_{-0.02}^{+0.04}$ & $0.15_{-0.03}^{+0.03}$ & $0.16_{-0.03}^{+0.02}$ & $0.20_{-0.04}^{+0.02}$ \\
\hline$R_{\text {in }}\left(R_{g}\right)$ & $4.71_{-0.88}^{+0.65}$ & $5.78_{-0.77}^{+0.43}$ & $6.61_{-0.37}^{+0.33}$ & $4.76_{-0.48}^{+0.28}$ & $6.73_{-0.73}^{+0.59}$ & $4.06_{-0.62}^{+0.32}$ & $4.88_{-0.91}^{+0.74}$ & $6.92_{-0.85}^{+0.65}$ \\
\hline$N_{\text {rel }}\left(10^{-5} \mathrm{ph} \mathrm{cm}^{-2} \mathrm{~s}^{-1}\right)$ & $0.62_{-0.10}^{+0.08}$ & $7.11_{-0.62}^{+0.42}$ & $0.49_{-0.07}^{+0.06}$ & $1.18_{-0.04}^{+0.03}$ & $1.87_{-0.23}^{+0.14}$ & $0.67_{-0.10}^{+0.07}$ & $1.10_{-0.06}^{+0.12}$ & $0.56_{-0.07}^{+0.04}$ \\
\hline$\chi^{2} / \mathrm{dof}$ & 983/992 & $2845 / 2550$ & $2275 / 2119$ & $1648 / 1640$ & $685 / 646$ & $829 / 828$ & $541 / 532$ & $533 / 557$ \\
\hline
\end{tabular}

$\xi$ 's are in the unit ergs $\mathrm{cm} \mathrm{s}^{-1} . \dagger$ pegged at the lowest value.

Table 7. Luminosities of NGC 1566 in the observations analyzed here.

\begin{tabular}{ccccccc}
\hline ID & Day & $\begin{array}{c}L_{\text {nuc }} \\
\left(10^{42} \mathrm{erg} \mathrm{s}^{-1}\right)\end{array}$ & $\begin{array}{c}L_{\text {soft }} \\
\left(10^{41} \mathrm{erg} \mathrm{s}^{-1}\right)\end{array}$ & $\begin{array}{c}L_{0.1-100} \\
\left(10^{42} \mathrm{erg} \mathrm{s}^{-1}\right)\end{array}$ & $\begin{array}{c}L_{\text {bol }} \\
\left(10^{43} \mathrm{erg} \mathrm{s}^{-1}\right)\end{array}$ & $\lambda_{\text {Edd }}$ \\
\hline X1 & 57331 & $0.34 \pm 0.01$ & $0.89 \pm 0.06$ & $0.43 \pm 0.01$ & $0.09 \pm 0.01$ & $0.003 \pm 0.001$ \\
O1 & 58295 & $13.42 \pm 0.08$ & $4.89 \pm 0.33$ & $13.91 \pm 0.08$ & $7.11 \pm 0.04$ & $0.066 \pm 0.001$ \\
O2 & 58395 & $4.02 \pm 0.05$ & $2.45 \pm 0.14$ & $4.26 \pm 0.05$ & $1.84 \pm 0.03$ & $0.017 \pm 0.001$ \\
O3 & 58639 & $2.36 \pm 0.01$ & $1.16 \pm 0.25$ & $2.48 \pm 0.03$ & $1.19 \pm 0.02$ & $0.011 \pm 0.001$ \\
O4 & 58703 & $4.01 \pm 0.05$ & $1.56 \pm 0.22$ & $4.17 \pm 0.05$ & $2.04 \pm 0.03$ & $0.019 \pm 0.001$ \\
O5 & 58706 & $2.16 \pm 0.06$ & $1.64 \pm 0.21$ & $2.32 \pm 0.06$ & $1.33 \pm 0.05$ & $0.012 \pm 0.001$ \\
O6 & 58713 & $1.96 \pm 0.12$ & $1.54 \pm 0.29$ & $2.11 \pm 0.12$ & $1.29 \pm 0.07$ & $0.012 \pm 0.001$ \\
O7 & 58716 & $2.40 \pm 0.03$ & $1.59 \pm 0.31$ & $2.58 \pm 0.04$ & $1.31 \pm 0.03$ & $0.012 \pm 0.001$ \\
\hline
\end{tabular}

$L_{\text {nuc }}$ and $L_{\text {soft }}$ are calculated for the primary power-law and soft excess components, respectively.

Eddington ratio, $\lambda_{\mathrm{Edd}}$ is calculated using $L_{\mathrm{bol}} / L_{\mathrm{Edd}}$ for a $\mathrm{BH}$ of mass $8.3 \times 10^{6} M_{\odot}$.

with the relativistic reflection model RELXILL to probe the reflection component.

Parker et al. (2019) analyzed O1 observation with RELXILL and XILLVER models in their spectral analysis. They found $\xi=10^{2.4 \pm 0.1}$ erg $\mathrm{cm}^{-2} \mathrm{~s}^{-1}, A_{\mathrm{Fe}}=3 \pm 0.2 A_{\odot}$ and $R_{\mathrm{refl}}=0.091 \pm 0.005$. In

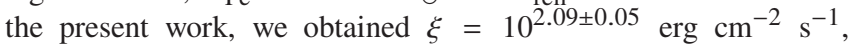
$A_{\mathrm{Fe}}=3.89 \pm 0.35$, and $R_{\text {refl }}=0.16 \pm 0.04$ for $\mathrm{O} 1$. The marginal difference between our results and those of Parker et al. (2019) could be ascribed to the different spectral models used. We observed fairly constant ionization $\left(\xi \sim 10^{1.9-2.2} \mathrm{erg} \mathrm{cm} \mathrm{s}^{-1}\right)$ and iron abundances $\left(A_{\mathrm{Fe}} \sim 4-5 A_{\odot}\right)$ across the observations. This is expected within our short period of observation. In all the spectra, we found a very weak reflection with reflection fraction, $R_{\text {refl }}<0.2$. We found a weak correlation between the reflection fraction $\left(R_{\text {refl }}\right)$ and the luminosity 

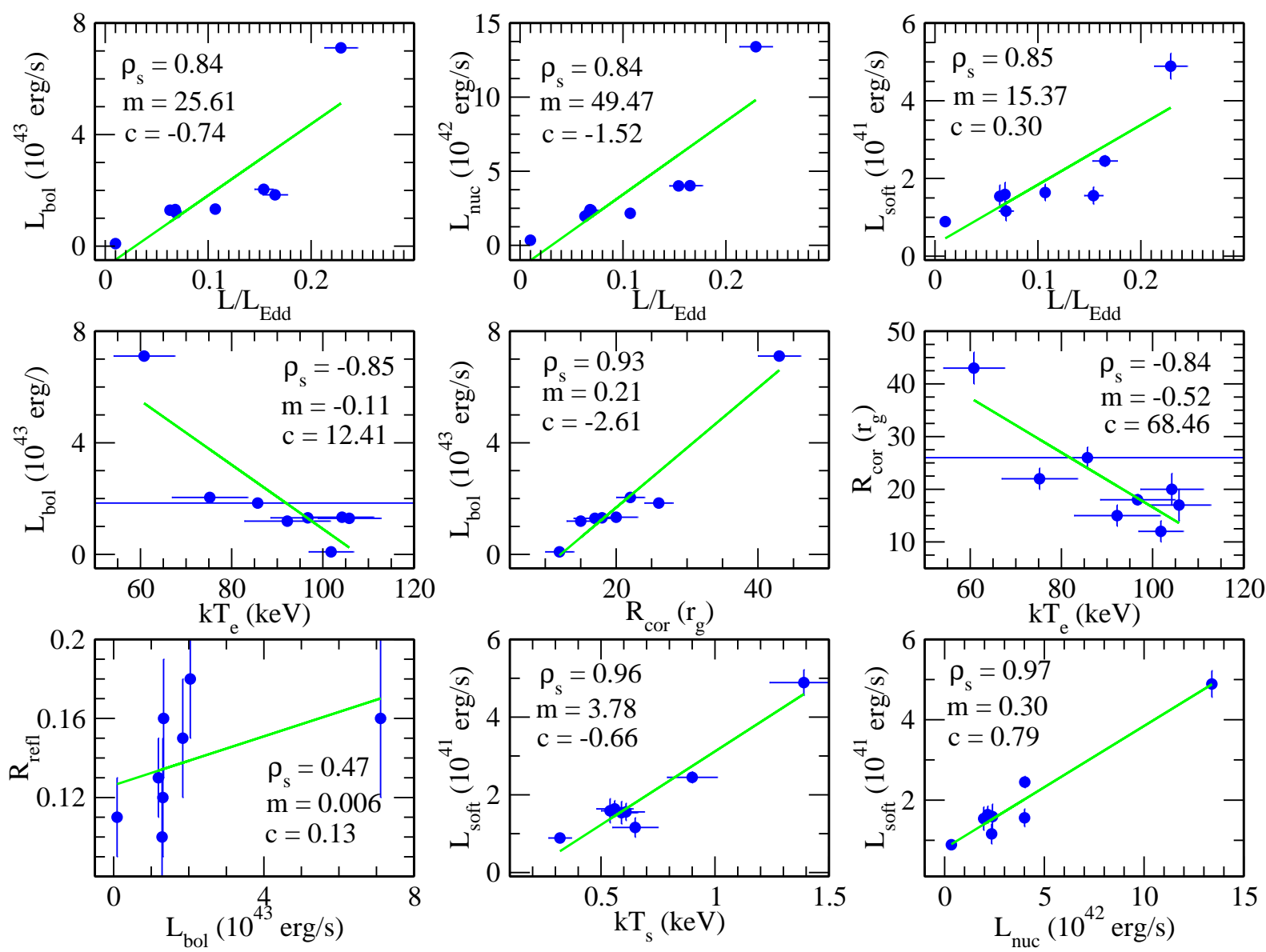

Figure 9. Correlation between different spectral parameters. In each panel, the corresponding Pearson correlation co-efficient $\left(\rho_{S}\right)$ is quoted. The solid green line in each panel represent the linear fit, $y=m x+c$. Corresponding fitted values of the slope $(\mathrm{m})$ and intercept (c) are also mentioned in each panels.

with the Pearson correlation coefficient of 0.47 . In general, reflection becomes strong with increase in the luminosity (Zdziarski et al. 1999). The low inclination angle of the source also results in a weak reflection (Ricci et al. 2011; Chatterjee et al. 2018). Therefore, the observed weak correlation between the reflection fraction and luminosity in NGC 1566 could be due to the low inclination angle of the source.

During the observations, a strong Fe $\mathrm{K} \alpha$ emission line with equivalent width $E W>100 \mathrm{eV}$ was detected, despite a weak reflection component, except for O5. This could be explained by high iron abundances in the reflector. From the spectral analysis with model4 , we found the inner edge of the disc extends up to $\sim 5 R_{\mathrm{g}}$. If iron originates from the inner disc, a broad iron line is expected. However, we did not observe a broad iron line. Either the broad line was absent or it was blurred beyond detection. However, during our observation, a narrow iron line was detected, which given its width, originates in the material further out than the accretion disc. Hence, from the full-width at half maximum (FWHM) of the line, we tried to constraint the $\mathrm{Fe} \mathrm{K} \alpha$ line emitting region. During our observation period, the FWHM of Fe K $\alpha$ line emission was found to be $<8700$ $\mathrm{km} \mathrm{s}^{-1}$, which corresponds to the region $>1200 R_{\mathrm{g}}$ from the $\mathrm{BH}$. This corresponds to the distance at which we expect to find the BLR (Kaspi et al. 2000). Thus, the BLR is the most probable Fe $\mathrm{K} \alpha$ line emitting region in NGC 1566.

\subsection{Soft Excess}

The origin of the soft excess in AGNs is still very debated, and several models have been proposed to explain it. Relativistic blurred ionized reflection from the accretion disc has been put forward as a likely explanation for the soft excess in many sources (Fabian et al. 2002; Ross \& Fabian 2005; Walton et al. 2013; García et al. 2019; Ghosh \& Laha 2020). An alternative scenario considers Comptonization by a optically thick, cold corona (Done et al. 2012). In this model, the comptonizing region is located above the inner accretion disc as a thin layer. Heating of circumnuclear region by bulk motion Comptonization in AGN with high Eddington ratio is also considered to be the reason for the soft excess (Kaufman et al. 2017). Recently, Nandi et al. (2021) argued from long-term observations and Monte-Carlo simulations (see Chatterjee et al. (2018) and references therein) that the thermal Comptonization of photons which have suffered fewer scatterings could explain the origin of soft excess in Ark 120.

In our analysis, we used a bbody component to take into account the soft excess. The blackbody temperature $\left(k T_{\mathrm{bb}}\right)$ was roughly constant with $k T_{\mathrm{bb}} \sim 110 \mathrm{eV}$ during our observations. This is consistent with the observation of other nearby AGNs (Gierliński \& Done 2004; Winter et al. 2009; Ricci et al. 2017; García et al. 2019). A good-fit with the OPTXAGNF model favoured soft Comptonization by an optically thick corona as the origin of the soft excess. During observation $\mathrm{O} 1$, the temperature of the optically-thick corona was 
observed to be $\sim 1.4 \mathrm{keV}$. Later, the optically thick corona cooled with decreasing luminosity.

We tried to estimate delay and correlation between the soft excess and X-ray continuum light curves (see Section 3.1.2). In the OPTXAGNF scenario, the total energetic depends on the mass accretion rate and is divided between the soft-Comptonization (softexcess) and hard-Comptonization (nuclear or primary emission). We found a strong correlation between the soft excess luminosity $\left(L_{\mathrm{soft}}\right)$ and nuclear luminosity $\left(L_{\text {nuc }}\right)$. The soft-excess luminosity and the Eddington ratio $\left(L / L_{\mathrm{Edd}}\right)$ were found to be correlated $\left(\rho_{\mathrm{S}}=0.78\right)$. This indicated that the soft excess strongly depended on the accretion rate, supporting the soft-Comptonization as the origin of the soft excess. We also found a delay of $\sim 10$ minutes between the soft excess and primary emission, indicating the origin of the soft excess was beyond the corona, possibly the accretion disc. Higher variability was also observed in the soft excess (see Section 3.1.2) during observations $\mathrm{X} 1, \mathrm{O} 1$, and $\mathrm{O} 3$. This could indicate a higher stochasticity in the origin of the soft-excess. It should be noted that, theoretically, infinite scattering produces blackbody which has the lowest variability. As Nandi et al. (2021) suggested, fewer scattering which could generate higher variability than the large number of scatterings, possibly lead to the origin of soft-excess. Overall, the soft excess in NGC 1566 has a complex origin, including reflection from the accretion disc and soft-Comptonization.

\subsection{Changing-Look Event and its Evolution}

The physical drivers of CL events are still highly debated, and could change from source to source. Tidal disruption events (TDEs), changes in obscuration, and variations in the mass accretion rate could all be possible explanations for CL events.

In the pre-outburst observation, $\mathrm{X} 1$, the absorber was not strongly ionized $\left(\xi_{1}<0.001\right)$, and had a column density of $N_{\mathrm{H}, 1}=$ $(3.53 \pm 0.06) \times 10^{21} \mathrm{~cm}^{-2}$. Two ionizing absorbers were detected during the observation $\mathrm{O} 1, \mathrm{O} 2$, and $\mathrm{O} 3$ which could be associated with an outflow. Parker et al. (2019) also observed two ionized absorbers during O1, and suggested that they could be associated with an outflow. The highest ionization (for both absorbers) was observed in O1, which corresponded to the epoch in which the observed luminosity was the highest. During the 2018 outburst, when the X-ray intensity increased, the emitted radiation may have caused the sublimation of the dust along the line of sight, thereby decreasing the hydrogen column density (Parker et al. 2019). As the X-ray intensity decreased after the main outburst, the dust could have condensed, leading to an increase in the column density. Eventually, during the August 2019 observations, the dust formation was stable as the hydrogen column density was approximately constant $\left(N_{\mathrm{H}} \sim 1.3 \times 10^{21} \mathrm{~cm}^{-2}\right)$. Generally, the dust clouds can recover in the timescale of several years (Kishimoto et al. 2013; Oknyansky et al. 2017). In this case, we observed that the dust clouds already recovered with an increase in $N_{\mathrm{H}}$ from $\sim 6 \times 10^{20} \mathrm{~cm}^{-2}$ to $1.3 \times 10^{21} \mathrm{~cm}^{-2}$ in $\sim 14$ months time. If this is correct, we would expect the $N_{\mathrm{H}}$ to reach at its pre-outburst value of $N_{\mathrm{H}} \sim 3.5 \times 10^{21} \mathrm{~cm}^{-2}$ in next few months.

The strong correlation between the accretion rate and the bolometric luminosity suggests that the accretion rate is responsible for the CL events in NGC 1566 during the 2018 outburst. Parker et al. (2019) also discussed several possibilities for the CL event in NGC 1566 and concluded that the disc-instability is the most likely reason for the outburst. The instability at the outer disc could propagate through the disc and cause the outburst. Noda \& Done (2018) explained the flux drop and changing-look phenomena in Mrk 1018 with the discinstability model where the time-scale for the changing-look event was $\sim 8$ years. The time-scale for changing-look event for NGC 1566 was $\sim 10$ months as the flux started to increase from September 2017 (Dai et al. 2018; Cutri et al. 2018). The time-scales are similar if we consider the mass of the BH. As the mass of Mrk 1018 is $M_{1018} \sim 10^{7.84} M_{\odot}$ (Ezhikode et al. 2017), the expected timescale for NGC 1566 is $\sim 8$ years/10 $\sim 10$ months. The observed ' $q$ '-shaped in the HID during the main outburst (F1), also suggest of disc instability as seen in the case of the outbursting black holes. The ' $q$ '-shaped HID is very common for the outbursting black holes (Remillard \& McClintock 2006) where disc instability is believed to lead the outburst. Noda \& Done (2018) also suggested that the soft-excess would decrease much more compared to the hard Xray with decreasing $L / L_{\text {Edd }}$ NGC 1566 also showed the strongest soft-excess emission during the highest $L / L_{\text {Edd }}(\mathrm{O} 1)$, while the softexcess emission dropped as $L / L_{\text {Edd }}$ decreased. In the pre-outburst quiescent state, no soft-excess was observed in NGC 1566. As the soft-excess can produce most of the ionizing photons necessary to create broad optical lines (Noda \& Done 2018), the broad line appeared during $\mathrm{O} 1$ (when the soft-excess was strong), leading to the changing-look event.

A TDE might be another possible explanation for the 2018 outburst of NGC 1566. A TDE could supply the accreting matter to the central SMBH, which would lead to an increase in luminosity. Several recurrent outbursts (F2, F3 \& F4) and nearly periodic X-ray variations were observed after the main outburst $(\mathrm{F} 1)$ as seen in the upper panel of Fig. 1 and Fig 10. In TDEs, some amount of matter could be left out, and cause recurrent outbursts (Komossa 2017). In the case of a classic TDE, after a star is tidally disrupted by the $\mathrm{SMBH}$, a decay profile of the luminosity with $t^{-5 / 3}$ is expected (Rees 1988; Komossa 2015, 2017), which is not observed in this case. During all observations, the source showed a relatively hard spectrum, with $\Gamma \sim 1.6-1.7$. This is clear contrast with classic TDEs, which typically show much softer spectra $(\Gamma \geq 3)$ (Komossa 2017). During the June 2018 outburst (F1), the X-ray luminosity changed by about $\sim 25$ times compared to the low state, which is low in comparison to other candidate TDEs. For example, 1ES 1927+654 and RX J1242-1119 showed a change in luminosity by over 4 orders of magnitude (Ricci et al. 2020) and 1500 times (Komossa et al. 2004), respectively. In general, no iron emission line is observed in the $\mathrm{X}$ ray band during the TDE (Saxton et al. 2020), whereas the case of NGC 1566 a strong $\mathrm{Fe} \mathrm{K} \alpha$ line was observed. Considering all this, we deem unlikely that the June 2018 outburst of NGC 1566 was triggered by a TDE.

An alternative explanation could be that a star is tidally disrupted by a merging SMBH binary at the center of NGC 1566 . According to Hayasaki \& Loeb (2016), after the stellar tidal disruption, the debris chaotically moves in the binary potential and is well-stretched. The debris orbital energy is then dissipated by the shock due to the self-crossing, leading to the formation of an accretion disc around each black hole after several mass exchanges through the Lagrange (L1) point of the binary system. If the orbital period of an unequalmass binary is short enough ( $<1000$ days) to lose the orbital energy by gravitational wave $(\mathrm{GW})$ emission, the secondary, less massive SMBH orbits around the center of mass at highly relativistic speed, while the primary SMBH hardly moves. Therefore, the electromagnetic emission from the accretion disc around the secondary black hole would be enhanced periodically by relativistic Doppler boosting. This scenario could explain the recurrent outburst observed in case of NGC 1566 (see Fig 10). From this figure, the orbital period is estimated to be $P_{\text {orb }} \sim 160$ days (between F1 \& F2). Taking into account the SMBH mass $\left(M_{\mathrm{BH}}=8.3 \times 10^{6} \mathrm{M}_{\odot}\right)$, we obtain $a \sim 710 r_{\mathrm{s}} \approx 5.6 \times 10^{-4} \mathrm{pc}$ as a binary semi-major axis, where 


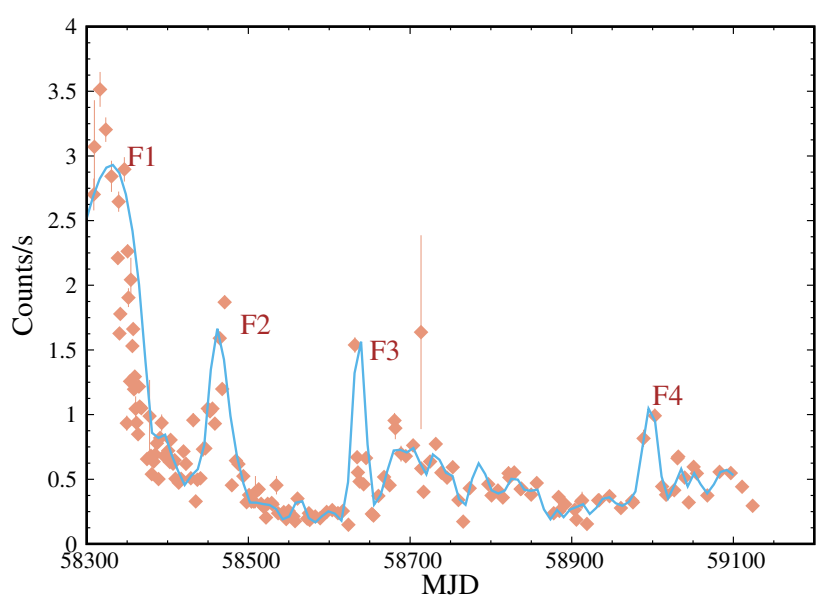

Figure 10. Lightcurve of NGC 1566 between June 2018 and August 2020 with smooth lines showing near-periodic variation of count rate.

$r_{\mathrm{s}}=2 G M_{\mathrm{BH}} / c^{2}$ is the Schwarzschild radius. The merging timescale of two SMBH with mass ratio $q$ due to $\mathrm{GW}$ emission is given by (Peters 1964),

$t_{\mathrm{gw}}=\frac{5}{8} \frac{(1-q)^{2}}{q} \frac{r_{s}}{c}\left(\frac{a}{r_{s}}\right) \sim 5.0 \times 10^{6} y r$.

This suggests that more than 100 TDEs could occur before the SMBH merger, considering the TDE rate for a single SMBH $\left(10^{-4}\right.$ to $10^{-5} y r^{-1}$ per galaxy, Stone et al. 2020). However, the event rate can be enhanced up to $0.1 y^{-1}$ per galaxy due to chaotic orbital evolution and Kozai-Lidov effect in the case of SMBH binaries (Chen et al. 2009; Li et al. 2015). Moreover, if stars are supplied by accretion from a circumbinary disc (eg, Hayasaki et al. 2007; Amaro-Seoane et al. 2013), then the TDE rate could be higher up to $\sim 0.2 \mathrm{yr}^{-1}$ if the mass supply rate is at the Eddington limit (Wolf et al. 2021). Therefore, the detection of similar, periodic burst events in the next few years to tens of years would support this interpretation.

\section{SUMMARY}

We analyzed the X-ray emission of the changing-look AGN NGC 1566 between 2015 and 2019. Our key findings are the following.

(i) NGC 1566 showed a giant outburst in June 2018 when the Xray luminosity increased by $\sim 25-30$ times compared to that during the low state. After the main outburst, several recurrent outbursts were also observed.

(ii) NGC 1566 hosts a low-spinning $\mathrm{BH}$ with the spin parameter, $a^{*} \sim 0.2$

(iii) The inclination angle is estimated to be in the range of $i \sim$ $10^{\circ}-21^{\circ}$.

(iv) The variation of the accretion rate is responsible for the evolution of the Compton corona and X-ray luminosity.

(v) A rise in the accretion rate is responsible for the change of luminosity. The HID or 'q'-diagram links the CL event of NGC 1566 with the outbursting black holes.

(vi) A strong soft excess was observed when the luminosity of NGC 1566 was high. The origin of the soft excess is observed to be complex. (vii) We rule out the possibility that the event was triggered by a classical TDE, where a star is tidally disrupted by the SMBH.

(viii) We propose a possible scenario where the central core is a merging binary SMBH. This scenario could explain the recurrent outburst.

\section{DATA AVAILABILITY}

We have used archival data for our analysis in this manuscript. All the models and software used in this manuscript are publicly available. Appropriate links are given in the text.

\section{ACKNOWLEDGEMENTS}

We acknowledge the anonymous reviewer for the helpful comments and suggestions which improved the paper. Work at Physical Research Laboratory, Ahmedabad, is funded by the Department of Space, Government of India. PN acknowledges CSIR fellowship for this work. A. C. and K.H. has been supported by the Basic Science Research Program through the National Research Foundation of Korea (NRF) funded by the Ministry of Education (2016R1A5A1013277 (A.C. and K.H) and 2020R1A2C1007219 (K.H.)). K.H. acknowledges the Yukawa Institute for Theoretical Physics (YITP) at Kyoto University. Discussions during the YITP workshop YITP-T19-07 on International Molecule-type Workshop "Tidal Disruption Events: General Relativistic Transients" were useful for this work. This research has made use of data and/or software provided by the High Energy Astrophysics Science Archive Research Center (HEASARC), which is a service of the Astrophysics Science Division at NASA/GSFC and the High Energy Astrophysics Division of the Smithsonian Astrophysical Observatory. This research has made use of the NuSTAR Data Analysis Software (NuSTARDAS) jointly developed by the ASI Space Science Data Center (SSDC, Italy) and the California Institute of Technology (Caltech, USA). This research has made use of the SIMBAD database, operated at CDS, Strasbourg, France.

\section{REFERENCES}

Alexander T., 1997, Is AGN Variability Correlated with Other AGN Properties? ZDCF Analysis of Small Samples of Sparse Light Curves. p. 163, doi:10.1007/978-94-015-8941-3_14

Alloin D., Pelat D., Phillips M., Whittle M., 1985, ApJ, 288, 205

Alloin D., Pelat D., Phillips M. M., Fosbury R. A. E., Freeman K., 1986, ApJ, 308,23

Amaro-Seoane P., Brem P., Cuadra J., 2013, ApJ, 764, 14

Antonucci R., 1993, ARA\&A, 31, 473

Aretxaga I., Joguet B., Kunth D., Melnick J., Terlevich R. J., 1999, ApJ, 519, L123

Arnaud K. A., 1996, in Jacoby G. H., Barnes J., eds, Astronomical Society of the Pacific Conference Series Vol. 101, Astronomical Data Analysis Software and Systems V. p. 17

Arnaud K. A., et al., 1985, MNRAS, 217, 105

Bennett C. L., et al., 2003, ApJS, 148, 1

Bianchi S., Piconcelli E., Chiaberge M., Bailón E. J., Matt G., Fiore F., 2009, ApJ, 695, 781

Braito V., Ballo L., Reeves J. N., Risaliti G., Ptak A., Turner T. J., 2013, MNRAS, 428, 2516

Chatterjee A., Chakrabarti S. K., Ghosh H., Garain S. K., 2018, MNRAS, 478,3356

Chen X., Madau P., Sesana A., Liu F. K., 2009, ApJ, 697, L149 
Cohen R. D., Rudy R. J., Puetter R. C., Ake T. B., Foltz C. B., 1986, ApJ, 311,135

Cutri R. M., Mainzer A. K., Dyk S. D. V., Jiang N., 2018, The Astronomer's Telegram, 11913, 1

Dai X., Stanek K. Z., Kochanek C. S., Shappee B. J., ASAS-SN Collaboration 2018, The Astronomer's Telegram, 11893, 1

Dauser T., Garcia J., Parker M. L., Fabian A. C., Wilms J., 2014, MNRAS, 444, L100

Dauser T., García J., Walton D. J., Eikmann W., Kallman T., McClintock J., Wilms J., 2016, A\&A, 590, A76

Denney K. D., et al., 2014, ApJ, 796, 134

Done C., Gierliński M., Kubota A., 2007, A\&ARv, 15, 1

Done C., Davis S. W., Jin C., Blaes O., Ward M., 2012, MNRAS, 420, 1848

Ducci L., Siegert T., Diehl R., Sanchez-Fernand ez C., Ferrigno C., Savchenko V., Bozzo E., 2018, The Astronomer's Telegram, 11754, 1

Edelson R., Malkan M., 2012, ApJ, 751, 52

Edelson R. A., et al., 1996, ApJ, 470, 364

Edelson R., Griffiths G., Markowitz A., Sembay S., Turner M. J. L., Warwick R., 2001, ApJ, 554, 274

Edelson R., Turner T. J., Pounds K., Vaughan S., Markowitz A., Marshall H., Dobbie P., Warwick R., 2002, ApJ, 568, 610

Elitzur M., 2012, ApJ, 747, L33

Elitzur M., Ho L. C., Trump J. R., 2014, MNRAS, 438, 3340

Elvis M., Risaliti G., Nicastro F., Miller J. M., Fiore F., Puccetti S., 2004, ApJ, 615, L25

Eracleous M., Halpern J. P., 2001, ApJ, 554, 240

Eracleous M., Livio M., Halpern J. P., Storchi-Bergmann T., 1995, ApJ, 438, 610

Evans P. A., et al., 2009, MNRAS, 397, 1177

Ezhikode S. H., Gandhi P., Done C., Ward M., Dewangan G. C., Misra R., Philip N. S., 2017, MNRAS, 472, 3492

Fabian A. C., Ballantyne D. R., Merloni A., Vaughan S., Iwasawa K., Boller T., 2002, MNRAS, 331, L35

Fabian A. C., Lohfink A., Kara E., Parker M. L., Vasudevan R., Reynolds C. S., 2015, MNRAS, 451, 4375

Ferrigno C., Siegert T., Sanchez-Fernandez C., Kuulkers E., Ducci L., Savchenko V., Bozzo E., 2018, The Astronomer's Telegram, 11783, 1

García J., et al., 2014, ApJ, 782, 76

García J. A., et al., 2019, ApJ, 871, 88

George I. M., Fabian A. C., 1991, MNRAS, 249, 352

Ghosh R., Laha S., 2020, MNRAS, 497, 4213

Gierliński M., Done C., 2004, MNRAS, 349, L7

Grupe D., Komossa S., Schartel N., 2018a, The Astronomer's Telegram, 11903, 1

Grupe D., et al., 2018b, The Astronomer's Telegram, 12314, 1

Grupe D., et al., 2019, The Astronomer's Telegram, 12826, 1

Guainazzi M., 2002, MNRAS, 329, L13

Guainazzi M., et al., 2016, MNRAS, 460, 1954

HI4PI Collaboration et al., 2016, A\&A, 594, A116

Haardt F., Maraschi L., 1991, ApJ, 380, L51

Harrison F. A., et al., 2013, ApJ, 770, 103

Hayasaki K., Loeb A., 2016, Scientific Reports, 6, 35629

Hayasaki K., Mineshige S., Sudou H., 2007, PASJ, 59, 427

Homan J., Wijnands R., van der Klis M., Belloni T., van Paradijs J., KleinWolt M., Fender R., Méndez M., 2001, ApJS, 132, 377

Jana A., Chatterjee A., Kumari N., Nandi P., Naik S., Patra D., 2020, MNRAS, 499, 5396

Jansen F., et al., 2001, A\&A, 365, L1

Kaspi S., Smith P. S., Netzer H., Maoz D., Jannuzi B. T., Giveon U., 2000, ApJ, 533, 631

Kaufman J., Blaes O. M., Hirose S., 2017, MNRAS, 467, 1734

Kishimoto M., et al., 2013, ApJ, 775, L36

Komossa S., 2015, Journal of High Energy Astrophysics, 7, 148

Komossa S., 2017, Astronomische Nachrichten, 338, 256

Komossa S., Halpern J., Schartel N., Hasinger G., Santos-Lleo M., Predehl P., 2004, ApJ, 603, L17

Korista K. T., Goad M. R., 2004, ApJ, 606, 749

Koss M., et al., 2017, ApJ, 850, 74
Kuin P., Bozzo E., Ferrigno C., Savchenko V., Kuulkers E., Ducci C. S.-F. L., Ducci L., 2018, The Astronomer's Telegram, 11786, 1

Li G., Naoz S., Kocsis B., Loeb A., 2015, MNRAS, 451, 1341

MacLeod C. L., et al., 2016, MNRAS, 457, 389

Makishima K., et al., 2000, ApJ, 535, 632

Matt G., Perola G. C., Piro L., 1991, A\&A, 247, 25

Matt G., Guainazzi M., Maiolino R., 2003, MNRAS, 342, 422

Merloni A., et al., 2015, MNRAS, 452, 69

Nandi P., Chatterjee A., Chakrabarti S. K., Dutta B. G., 2021, MNRAS,

Nandra K., George I. M., Mushotzky R. F., Turner T. J., Yaqoob T., 1997, ApJ, 476, 70

Nardini E., Risaliti G., 2011, MNRAS, 417, 2571

Nenkova M., Sirocky M. M., Ivezić Ž., Elitzur M., 2008a, ApJ, 685, 147

Nenkova M., Sirocky M. M., Nikutta R., Ivezić Ž., Elitzur M., 2008b, ApJ, 685,160

Netzer H., 2015, ARA\&A, 53, 365

Nicastro F., 2000, ApJ, 530, L65

Noda H., Done C., 2018, MNRAS, 480, 3898

Ochmann M. W., Kollatschny W., Zetzl M., 2020, Contributions of the Astronomical Observatory Skalnate Pleso, 50, 318

Oknyansky V. L., et al., 2017, MNRAS, 467, 1496

Oknyansky V. L., Lipunov V. M., Gorbovskoy E. S., Winkler H., van Wyk F., Tsygankov S., Buckley D. A. H., 2018, The Astronomer's Telegram, 11915,1

Oknyansky V. L., Winkler H., Tsygankov S. S., Lipunov V. M., Gorbovskoy E. S., van Wyk F., Buckley D. A. H., Tyurina N. V., 2019, MNRAS, 483,558

Oknyansky V. L., et al., 2020, MNRAS, 498, 718

Padovani P., et al., 2017, A\&ARv, 25, 2

Parker M. L., et al., 2019, MNRAS, 483, L88

Pastoriza M., Gerola H., 1970, Astrophys. Lett., 6, 155

Peters P. C., 1964, Physical Review, 136, 1224

Piconcelli E., Bianchi S., Guainazzi M., Fiore F., Chiaberge M., 2007, A\&A, 466,855

Quintana H., Kaufmann P., Sasic J. L., 1975, MNRAS, 173, 57P

Rees M. J., 1988, Nature, 333, 523

Remillard R. A., McClintock J. E., 2006, ARA\&A, 44, 49

Ricci C., Walter R., Courvoisier T. J. L., Paltani S., 2011, A\&A, 532, A102

Ricci C., et al., 2016, ApJ, 820, 5

Ricci C., et al., 2017, ApJS, 233, 17

Ricci C., et al., 2018, MNRAS, 480, 1819

Ricci C., et al., 2020, ApJ, 898, L1

Ricci C., et al., 2021, ApJS, 255, 7

Risaliti G., Elvis M., Nicastro F., 2002, ApJ, 571, 234

Risaliti G., Elvis M., Fabbiano G., Baldi A., Zezas A., Salvati M., 2007, ApJ, 659, L111

Ross R. R., Fabian A. C., 2005, MNRAS, 358, 211

Runnoe J. C., et al., 2016, MNRAS, 455, 1691

Saxton R., Komossa S., Auchettl K., Jonker P. G., 2020, Space Sci. Rev., 216,85

Shakura N. I., Sunyaev R. A., 1973, A\&A, 500, 33

Shappee B. J., et al., 2014, ApJ, 788, 48

Sheng Z., Wang T., Jiang N., Yang C., Yan L., Dou L., Peng B., 2017, ApJ, $846, \mathrm{~L} 7$

Shobbrook R. R., 1966, MNRAS, 131, 365

Sikora M., Stawarz Ł., Lasota J.-P., 2007, ApJ, 658, 815

Singh K. P., Garmire G. P., Nousek J., 1985, ApJ, 297, 633

Stone N. C., Vasiliev E., Kesden M., Rossi E. M., Perets H. B., Amaro-Seoane P., 2020, Space Sci. Rev., 216, 35

Strüder L., et al., 2001, A\&A, 365, L18

Sunyaev R. A., Titarchuk L. G., 1980, A\&A, 500, 167

Vasudevan R. V., Fabian A. C., 2009, MNRAS, 392, 1124

Vaughan S., Edelson R., Warwick R. S., Uttley P., 2003, MNRAS, 345, 1271

Verner D. A., Ferland G. J., Korista K. T., Yakovlev D. G., 1996, ApJ, 465, 487

Walton D. J., Nardini E., Fabian A. C., Gallo L. C., Reis R. C., 2013, MNRAS, 428, 2901

Wilms J., Allen A., McCray R., 2000, ApJ, 542, 914

Winkler H., 1992, MNRAS, 257, 677 
Winter L. M., Mushotzky R. F., Reynolds C. S., Tueller J., 2009, ApJ, 690,1322

Wolf J., et al., 2021, A\&A, 647, A5

Woo J.-H., Urry C. M., 2002, ApJ, 579, 530

Wu X.-B., Liu F. K., 2004, ApJ, 614, 91

Yaqoob T., Tatum M. M., Scholtes A., Gottlieb A., Turner T. J., 2015, MNRAS, 454, 973

Zdziarski A. A., Johnson W. N., Magdziarz P., 1996, MNRAS, 283, 193

Zdziarski A. A., Lubiński P., Smith D. A., 1999, MNRAS, 303, L11

Życki P. T., Done C., Smith D. A., 1999, MNRAS, 309, 561

da Silva P., Steiner J. E., Menezes R. B., 2017, MNRAS, 470, 3850

de Vaucouleurs G., 1973, ApJ, 181, 31

de Vaucouleurs G., de Vaucouleurs A., 1961, Mem. RAS, 68, 69

This paper has been typeset from a $\mathrm{T}_{\mathrm{E}} \mathrm{X} / \mathrm{L} \mathrm{T} \mathrm{E} \mathrm{X}$ file prepared by the author. 
Table 1. Swift/XRT observation log

\begin{tabular}{|c|c|c|c|c|c|c|c|c|}
\hline Obs ID & Date & $\operatorname{Exp}(\mathrm{s})$ & Obs ID & Date & $\operatorname{Exp}(\mathrm{s})$ & Obs ID & Date & $\operatorname{Exp}(\mathrm{s})$ \\
\hline 00035880002 & $2007-12-12$ & 2151 & 00035880060 & 2018-10-19 & 677 & 00035880122 & 2019-06-02 & 709 \\
\hline 00035880003 & 2008-01-02 & 3230 & 00035880061 & $2018-10-22$ & 967 & 00035880123 & 2019-06-03 & 694 \\
\hline 00045604001 & 2011-08-25 & 378 & 00035880062 & $2018-10-25$ & 934 & 00035880125 & 2019-06-04 & 519 \\
\hline 00045604002 & 2011-11-03 & 1135 & 00035880063 & $2018-10-28$ & 1135 & 00035880126 & 2019-06-05 & 909 \\
\hline 00045604003 & 2012-01-06 & 142 & 00035880064 & $2018-10-31$ & 937 & 00035880127 & 2019-06-06 & 754 \\
\hline 00045604004 & 2012-09-15 & 1210 & 00035880065 & 2018-11-06 & 957 & 00035880128 & 2019-06-07 & 659 \\
\hline 00045604005 & 2012-09-29 & 2773 & 00035880066 & 2018-11-09 & 1017 & 00035880129 & 2019-06-08 & 674 \\
\hline 00045604006 & 2012-09-29 & 4518 & 00035880067 & 2018-11-12 & 992 & 00035880130 & 2019-06-09 & 674 \\
\hline 00045604007 & 2012-10-09 & 4526 & 00035880068 & $2018-11-15$ & 917 & 00035880131 & 2019-06-10 & 814 \\
\hline 00045604008 & $2012-10-10$ & 1138 & 00035880069 & 2018-11-18 & 900 & 00035880132 & 2019-06-11 & 764 \\
\hline 00035880004 & 2018-06-24 & 1352 & 00035880070 & $2018-11-21$ & 822 & 00035880133 & 2019-06-13 & 369 \\
\hline 00035880005 & 2018-07-10 & 95 & 00035880071 & 2018-11-24 & 992 & 00035880134 & 2019-06-14 & 974 \\
\hline 00035880006 & 2018-07-10 & 1030 & 00035880072 & $2018-11-27$ & 1113 & 00035880136 & 2019-06-16 & 1048 \\
\hline 00035880007 & $2018-07-17$ & 254 & 00035880073 & $2018-11-30$ & 1119 & 00035880137 & 2019-06-19 & 1053 \\
\hline 00035880008 & 2018-07-17 & 999 & 00035880074 & $2018-12-03$ & 909 & 00035880138 & 2019-06-20 & 920 \\
\hline 00035880009 & $2018-07-24$ & 239 & 00035880075 & $2018-12-06$ & 1024 & 00035880139 & 2019-06-26 & 543 \\
\hline 00035880010 & $2018-07-24$ & 1059 & 00035880076 & $2018-12-12$ & 1084 & 00035880140 & 2019-07-03 & 892 \\
\hline 00035880013 & 2018-07-31 & 1073 & 00035880077 & $2018-12-15$ & 954 & 00035880141 & 2019-07-10 & 915 \\
\hline 00035880014 & 2018-08-08 & 975 & 00035880078 & $2018-12-18$ & 999 & 00035880142 & 2019-07-17 & 1103 \\
\hline 00035880015 & 2018-08-09 & 1205 & 00035880079 & $2018-12-21$ & 994 & 00035880143 & 2019-07-24 & 878 \\
\hline 00035880016 & 2018-08-10 & 969 & 00035880080 & $2018-12-24$ & 909 & 00035880144 & 2019-07-31 & 942 \\
\hline 00035880017 & $2018-08-11$ & 969 & 00035880081 & $2018-12-27$ & 584 & 00088910001 & 2019-08-08 & 1924 \\
\hline 00035880019 & $2018-08-13$ & 1009 & 00035880082 & $2018-12-31$ & 1079 & 00088910002 & 2019-08-18 & 1676 \\
\hline 00035880021 & $2018-08-16$ & 499 & 00035880083 & 2019-01-01 & 534 & 00088910003 & 2019-08-21 & 1960 \\
\hline 00035880022 & 2018-08-19 & 349 & 00035880084 & 2019-01-05 & 566 & 00045604010 & 2019-08-29 & 559 \\
\hline 00035880023 & $2018-08-20$ & 979 & 00035880085 & 2019-01-11 & 862 & 00045604011 & 2019-09-05 & 952 \\
\hline 00035880025 & $2018-08-21$ & 748 & 00035880086 & 2019-01-14 & 987 & 00045604012 & 2019-09-12 & 822 \\
\hline 00035880026 & $2018-08-23$ & 969 & 00035880087 & 2019-01-17 & 1007 & 00045604013 & 2019-09-19 & 839 \\
\hline 00035880027 & $2018-08-24$ & 1069 & 00035880088 & 2019-01-20 & 993 & 00045604014 & 2019-09-26 & 912 \\
\hline 00035880028 & $2018-08-25$ & 944 & 00035880089 & 2019-01-23 & 1064 & 00045604015 & $2019-10-03$ & 511 \\
\hline 00035880029 & $2018-08-26$ & 1044 & 00035880090 & 2019-01-26 & 1020 & 00045604016 & 2019-10-10 & 920 \\
\hline 00035880030 & $2018-08-27$ & 969 & 00035880091 & 2019-01-29 & 1020 & 00045604017 & 2019-10-17 & 997 \\
\hline 00035880031 & $2018-08-28$ & 1189 & 00035880092 & 2019-02-02 & 396 & 00045604020 & 2019-11-09 & 1313 \\
\hline 00035880032 & $2018-08-29$ & 1034 & 00035880094 & 2019-02-05 & 676 & 00045604021 & 2019-11-14 & 646 \\
\hline 00035880033 & $2018-08-30$ & 1009 & 00035880095 & 2019-02-07 & 1004 & 00045604022 & $2019-11-21$ & 738 \\
\hline 00035880034 & $2018-08-31$ & 939 & 00035880096 & 2019-02-10 & 987 & 00045604023 & $2019-11-28$ & 463 \\
\hline 00035880035 & 2018-09-01 & 959 & 00035880097 & 2019-02-14 & 1090 & 00045604024 & 2019-12-05 & 890 \\
\hline 00035880036 & 2018-09-02 & 959 & 00035880098 & 2019-02-16 & 1213 & 00045604025 & $2019-12-12$ & 1075 \\
\hline 00035880037 & 2018-09-03 & 1019 & 00035880099 & 2019-02-20 & 225 & 00045604026 & 2019-12-20 & 1217 \\
\hline 00035880038 & 2018-09-04 & 1284 & 00035880100 & 2019-02-23 & 888 & 00045604027 & $2020-01-01$ & 668. \\
\hline 00035880039 & 2018-09-04 & 984 & 00035880101 & 2019-03-01 & 947 & 00045604028 & 2020-01-08 & 1118 \\
\hline 00035880040 & 2018-09-05 & 984 & 00035880102 & 2019-03-04 & 1010 & 00045604031 & $2020-01-29$ & 669 \\
\hline 00035880041 & 2018-09-06 & 1059 & 00035880103 & 2019-03-07 & 1007 & 00045604032 & $2020-02-05$ & 699 \\
\hline 00035880042 & 2018-09-13 & 909 & 00035880104 & 2019-03-10 & 1183 & 00045604033 & 2020-02-12 & 870 \\
\hline 00035880043 & 2018-09-17 & 55 & 00035880105 & 2019-03-13 & 997 & 00045604035 & 2020-02-24 & 1061 \\
\hline 00035880044 & 2018-09-18 & 1329 & 00035880106 & 2019-03-16 & 862 & 00045604036 & $2020-02-26$ & 648 \\
\hline 00035880045 & 2018-09-19 & & 00035880107 & 2019-03-19 & & 00045604037 & 2020-03-04 & 950 \\
\hline 00035880046 & 2018-09-21 & 1000 & 00035880108 & 2019-03-30 & 724 & 00045604038 & $2020-03-11$ & 458 \\
\hline 00035880047 & $2018-09-23$ & 1013 & 00035880109 & 2019-04-02 & 822 & 00045604040 & $2020-03-25$ & 496 \\
\hline 00035880048 & $2018-09-25$ & 607 & 00035880110 & 2019-04-09 & 679 & 00045604039 & $2020-03-25$ & 528 \\
\hline 00035880049 & 2018-09-27 & 966 & 00035880111 & 2019-04-16 & 1012 & 00045604041 & 2020-04-08 & 922 \\
\hline 00035880050 & 2018-09-29 & 965 & 00035880112 & 2019-04-23 & 1002 & 00045604042 & $2020-04-22$ & 848 \\
\hline 00035880051 & 2018-10-01 & 992 & 00035880113 & 2019-04-30 & 371 & 00045604043 & 2020-05-06 & 781 \\
\hline 00035880053 & 2018-10-04 & 2139 & 00035880114 & 2019-05-07 & 684 & 00045604044 & $2020-05-20$ & 531 \\
\hline 00035880054 & $2018-10-08$ & 1064 & 00035880115 & 2019-05-14 & 1063 & 00045604045 & $2020-06-03$ & 1060 \\
\hline 00035880055 & 2018-10-09 & 989 & 00035880116 & 2019-05-21 & 1055 & 00045604046 & $2020-06-17$ & 632 \\
\hline 00035880056 & $2018-10-11$ & 986 & 00035880117 & 2019-05-28 & 1012 & 00045604047 & 2020-07-01 & 1769 \\
\hline 00035880057 & 2018-10-13 & 967 & 00035880119 & 2019-05-29 & 1015 & 00045604048 & $2020-07-15$ & 170 \\
\hline 00035880058 & $2018-10-15$ & 1125 & 00035880120 & 2019-05-31 & 699 & 00045604049 & $2020-07-20$ & 788 \\
\hline 00035880059 & $2018-10-17$ & 1269 & 00035880121 & 2019-06-01 & 999 & & & \\
\hline
\end{tabular}

